\title{
Infinite Dimensional Grassmannian Structure of Two-Dimensional Quantum Gravity
}

\author{
Masafumi Fukuma $^{1, \star}$, Hikaru Kawai ${ }^{1, \star \star}$, and Ryuichi Nakayama ${ }^{2, \star \star \star}$ \\ 1 Department of Physics, University of Tokyo, Bunkyo-ku, Tokyo 113, Japan \\ 2 National Laboratory for High Energy Physics (KEK), Tsukuba-shi, Ibaraki 305, Japan
}

Received March 22, 1991

\begin{abstract}
We study the infinite dimensional Grassmannian structure of 2D quantum gravity coupled to minimal conformal matters, and show that there exists a large symmetry, the $W_{1+\infty}$ symmetry. Using this symmetry structure, we prove that the square root of the partition function, which is a $\tau$ function of the $p$-reduced KP hierarchy, satisfies the vacuum condition of the $W_{1+\infty}$ algebra. We further show that this condition is reduced to the vacuum condition of the $W_{p}$ algebra when the redundant variables for the $p$-reduction are eliminated. This mechanism also gives a prescription for extracting the $W_{p}$ algebra from the $W_{1+\infty}$ algebra.
\end{abstract}

\section{Introduction}

Recently great progress has been made in the understanding of the nonperturbative aspects of $2 \mathrm{D}$ quantum gravity by formulating it in terms of the matrix models [1]. In particular, it has been recognized that the 2D quantum gravity has a close connection with the KP hierarchy [2-4]. Furthermore, the analysis through the Schwinger-Dyson equation reveals the universal structures of 2D quantum gravity and from this one can obtain an analogue of the operator product expansion even in the theory of quantum gravity $[5,6]$. In particular, it is shown $[5,6]$ that the Schwinger-Dyson equation for the 2D gravity coupled to the $(p, q)$ conformal matters $((p, q) \text { quantum gravity })^{1}$ is expressed as the vacuum condition of the $W_{p}$ algebra on the function $\tau(x)=\sqrt{Z(x)}$, where $Z(x)$ is the partition function of the 2D gravity with the action $S=\sum_{n \neq 0(\bmod p)} x_{n} \mathcal{O}_{n}$ and the $x$ 's

\footnotetext{
* E-mail address: tkyvax\$hepnet::fukuma

$\star \star$ E-mail address: tkyvax\$hepnet::kawai

$\star \star \star$ E-mail address: nakayama@jpnkekvm.bitnet

1 We use the convention different from that in [4] where the roles of $p$ and $q$ are interchanged
} 
control the renormalization group flows corresponding to the change of the parameter $q$.

Although this formulation by the Schwinger-Dyson equation gives a systematic description, it is not completely satisfactory for the purpose of revealing the universal structures of the 2D quantum gravity. In fact, in contrast to the case of the $q$ flows which we can easily control by varying the location of the background sources $x_{n}$, we must change the form of the constraint itself in order to control the $p$ flows. For example, while two theories parametrized by $(p, q)=(2,3)$ and $(p, q)$ $=(3,2)$ should be identical, the relation between two sets of the scaling operators $\left\{\mathcal{O}_{n}\right\}_{n \neq 0(\bmod 2)}$ and $\left\{\mathcal{O}_{n}\right\}_{n \neq 0(\bmod 3)}$ is not clear. Thus we need a more general framework for the 2D quantum gravity where the two parameters $p$ and $q$ can be changed freely.

In this paper, as a first step towards this direction, we investigate the symmetry structure of the 2D quantum gravity and show the existence of a large symmetry, the $W_{1+\infty}$ symmetry [7]. Using this symmetry structure, one can easily prove that the $\tau$ function automatically satisfies the vacuum condition of the $W_{1+\infty}$ algebra if it obeys the string equation. Furthermore, we show that this condition is reduced to the vacuum condition of the $W_{p}$ algebra when some redundant variables are eliminated. In this sense, the $W_{p}$ symmetry in the $(p, q)$ quantum gravity can be regarded as a by-product of the $W_{1+\infty}$ symmetry. Although we have not yet found a final framework in which the $p$ flows can be controlled, we expect that the exploration along this line leads to the final answer.

This paper is organized as follows. In Sect. 2, we summarize the Sato theory that describes the KP equation in terms of the infinite dimensional Grassmann manifold, and show how the string equations are treated in this formalism. In Sect. 3, after explaining the rule for translating the operators acting on the $\tau$ function into the one-body operators acting on the one-body wave function, we introduce the $w_{1+\infty}$ algebra as a set of the one-body differential operators. Then we show that the string equation takes a simple form when it is expressed in terms of the universal Grassmann manifold. In Sect. 4, we show that this $w_{1+\infty}$ algebra becomes its central extension, the $W_{1+\infty}$ algebra, when it is represented as a set of differential operators acting on the $\tau$ function. Furthermore, we prove that a $\tau$ function of the $p$-reduced KP hierarchy satisfies the vacuum condition of the $W_{1+\infty}$ algebra when it obeys the string equation. This form of the vacuum condition includes the variables, $x_{p}, x_{2 p}, \ldots$, which become redundant when we take the conditions of the $p$-reduction into account. In Sect. 5, we then show that after the elimination of these redundant variables the vacuum condition of the $W_{1+\infty}$ algebra is reduced to the vacuum condition of the $W_{p}$ algebra with central charge $c=p-1$. Section 6 is for some concluding remarks. In Appendix A, for the sake of completeness, we explain the relation between the Lax operator and the $\tau$ function. Appendices B and C are devoted to the details of the calculations in Sect. 5.

\section{The $\tau$ Functions of the KP Hierarchy and the String Equations}

In this section, we summarize the Sato theory that describes the KP equation in terms of the infinite dimensional Grassmann manifold [8-10], and show how the string equations are treated in this formalism.

Let $H$ be the set of formal Laurent series:

$$
H=\mathbf{C}\left[z, z^{-1}\right]=\left\{f(z)=\sum_{r \in \mathbf{Z}+1 / 2} a_{r} z^{-r-1 / 2}\right\}
$$


and $V$ be a subspace of $H$ which is spanned by linearly independent functions $\xi^{(i)}(z)$ $=\sum_{r \in \mathbf{Z}+1 / 2} \xi_{r}^{(i)} z^{-r-1 / 2}(i=0,1,2, \ldots)$ in $H ; V=\left[\xi^{(0)}(z), \xi^{(1)}(z), \xi^{(2)}(z), \ldots\right]$. We call $V$ comparable when $V$ is linearly isomorphic to the subspace $H_{-}=\left\{f(z)=\sum_{r<0} a_{r} z^{-r-1 / 2}\right\}$, and the universal Grassmann manifold (UGM) is defined as the set of such comparable subspaces:

$$
\mathrm{UGM}=\left\{V \subset H \mid V \simeq H_{-}\right\} .
$$

See, for example, [10] for more mathematically complete definitions.

In order to express the $\tau$ functions in a compact form, we introduce fermionic operators $\psi_{r}^{\dagger}, \psi_{s}(r, s \in \mathbf{Z}+1 / 2)$ satisfying the following anticommutation relations

$$
\left\{\psi_{r}^{\dagger}, \psi_{s}\right\}=\delta_{r s},\left\{\psi_{r}^{\dagger}, \psi_{s}^{\dagger}\right\}=0,\left\{\psi_{r}, \psi_{s}\right\}=0,
$$

and define the zero-particle state $|-\infty\rangle$ as the state that satisfies

$$
\psi_{r}|-\infty\rangle=0 \quad\left({ }^{\forall} r \in \mathbf{Z}+1 / 2\right) .
$$

We then make a correspondence between a vector $\xi(z)$ in $H$ and a fermionic operator $\psi[\xi]^{\dagger}$ in the following way:

$$
\xi(z)=\sum_{r \in \mathbf{Z}+1 / 2} \xi_{r} z^{-r-1 / 2} \leftrightarrow \psi[\xi]^{\dagger}=\sum_{r \in \mathbf{Z}+1 / 2} \xi_{r} \psi_{r}^{\dagger} .
$$

Furthermore, we associate a comparable subspace $V=\left[\xi^{(0)}(z), \xi^{(1)}(z), \ldots\right]$ of $H$ with a decomposable multi-fermion state $|g\rangle$ via $^{2}$

$$
V=\left[\xi^{(0)}(z), \xi^{(1)}(z), \ldots\right] \rightarrow|g\rangle=\psi\left[\xi^{(0)}\right]^{\dagger} \psi\left[\xi^{(1)}\right]^{\dagger} \ldots|-\infty\rangle .
$$

Obviously this correspondence between the UGM and the set of all decomposable states is one-to-one up to an overall factor. We denote the subspace $V$ associated with the decomposable state $|g\rangle$ by $V_{g}$. We further define the vacuum as the state of Dirac sea filled up to $r=-1 / 2$ and denote it by $|0\rangle$ :

$$
|0\rangle \equiv \psi_{-1 / 2}^{\dagger} \psi_{-3 / 2}^{\dagger} \cdots|-\infty\rangle \text {. }
$$

This state satisfies $\psi_{r}|0\rangle=0(r>0)$ and $\psi_{r}^{\dagger}|0\rangle=0(r<0)$.

The $\tau$ function is now defined as follows. First, we introduce the normal ordering for fermionic operators by

$$
: \psi_{r}^{\dagger} \psi_{s}: \equiv \begin{cases}\psi_{r}^{\dagger} \psi_{s} & (s>0) \\ -\psi_{s} \psi_{r}^{\dagger} & (s<0)\end{cases}
$$

and define the current operators $J_{n}$ as

$$
J_{n} \equiv \sum_{r}: \psi_{r-n}^{\dagger} \psi_{r}: \quad(n \in \mathbf{Z})
$$

or equivalently

$$
J(z)=\sum_{n \in \mathbf{Z}} J_{n} z^{-n-1}=: \psi^{\dagger}(z) \psi(z):,
$$

where $\psi(z)=\sum_{r \in \mathbf{Z}+1 / 2} \psi_{r} z^{-r-1 / 2}$ and $\psi^{\dagger}(z)=\sum_{r \in \mathbf{Z}+1 / 2} \psi_{-r}^{\dagger} z^{-r-1 / 2}$. Then, the $\tau$ func-

${ }^{2}$ A multi-fermion state $|g\rangle$ is called decomposable if it can be expressed in the form of the righthand side of Eq. (2.6). This state is nothing but the one that can be written as a Slater determinant 
tion associated with a decomposable state $|g\rangle$ is defined as a function of infinitely many variables $x=\left(x_{1}, x_{2}, x_{3}, \ldots\right)$ :

$$
\tau(x)=\left\langle 0\left|\exp \left(\sum_{n \geqq 1} x_{n} J_{n}\right)\right| g\right\rangle .
$$

Hereafter we will consider only neutral states, that is, $J_{0}|g\rangle=0$.

Note that this function $\tau(x)$ can be reinterpreted as the bosonic coherent representation of the state $|g\rangle$. In fact, if we introduce a free boson $\phi(z)$ via

$$
\partial \phi(z)=J(z)=: \psi^{\dagger}(z) \psi(z):
$$

or conversely

$$
\begin{gathered}
\psi^{\dagger}(z)=: e^{\phi(z)}:, \\
\psi(z)=: e^{-\phi(z)}:
\end{gathered}
$$

then $\langle 0| \exp \left(\sum_{n \geqq 1} x_{n} J_{n}\right)$ is nothing but the coherent state of the free boson. Thus, the following relations hold:

$$
\langle 0| e^{\sum_{n=1}^{\sum} x_{n} J_{n}} J_{m}=\left\{\begin{array}{ll}
\partial_{m}\langle 0| e^{\sum_{n=1} x_{n} J_{n}} & (m>0) \\
|m| x_{|m|}\langle 0| e^{n_{n} \geqq 1} x_{n} J_{n} & (m<0)
\end{array} .\right.
$$

The p-reduction of the KP hierarchy is defined by imposing the following additional constraint on the state $|g\rangle$ :

$$
J_{n p}|g\rangle=0 \quad(n=1,2,3, \ldots) .
$$

Due to (2.15), Eq. (2.16) is equivalent to

$$
\partial_{n p} \tau(x)=0 \quad(n=1,2,3, \ldots) .
$$

Now let us consider the following differential operators

$$
\mathscr{L}_{n} \equiv \frac{1}{2} \sum_{k+l=-n} k l x_{k} x_{l}+\sum_{k-l=-n} k x_{k} \partial_{l}+\frac{1}{2} \sum_{k+l=n} \partial_{k} \partial_{l},
$$

which satisfy the Virasoro algebra with central charge $c=1$ :

$$
\left[\mathscr{L}_{n}, \mathscr{L}_{m}\right]=(n-m) \mathscr{L}_{n+m}+\frac{1}{12}\left(n^{3}-n\right) \delta_{n+m, 0} .
$$

We then impose the following condition on a $\tau$ function of the $p$-reduced KP hierarchy ${ }^{3}$

$$
\mathscr{L}_{-p} \tau(x)=0 .
$$

This $\tau$ function can be identified with the square root of the partition function $Z(x)$ of the $(p, q)$ quantum gravity with source terms $\sum_{n \neq 0(\bmod p)} x_{n} \mathcal{O}_{n}$ :

$$
Z(x)=\tau(x)^{2} .
$$

\footnotetext{
3 Note that $x_{p}, x_{2 p}, x_{3 p}, \ldots$ are included in Eq. (2.18), which are redundant parameters under the $p$-reduction. However, in Eq. (2.20) they appear in the form $2 p x_{2 p} \partial_{p}+3 p x_{3 p} \partial_{2 p}+\ldots$, so that we can neglect them in Eq. (2.20). This kind of remark should be kept in mind in the following discussions
} 
One way to see this is to expand Eq. (2.20) around a background source

$$
x_{1}^{0}=t, x_{p+q}^{0}=\text { const, } x_{\text {others }}^{0}=0 \text {. }
$$

Using the equations in (A.2.17), one can show that the corresponding pseudodifferential operator $L$ for this background satisfies [5]

$$
\begin{gathered}
\left(L^{q}\right)_{-1}=\ldots=\left(L^{q}\right)_{-(p-2)}=0, \\
\left(L^{q}\right)_{-(p-1)}=\text { const } \cdot x_{1},
\end{gathered}
$$

which leads to the string equation of the $2 \mathrm{D}$ gravity coupled to $(p, q)$ minimal conformal matters [4]:

$$
\begin{aligned}
{\left[L^{p},\left(L^{q}\right)_{+}\right] } & =\text {const }, \\
\left(L^{p}\right)_{-} & =0 .
\end{aligned}
$$

Furthermore, as we will show in Sect. 4 in a more general framework, if a $\tau$ function of the $p$-reduced KP hierarchy satisfies the condition (2.20), then it automatically satisfies the Virasoro constraint

$$
\left\{\mathscr{L}_{n p}+\frac{1}{24}\left(p^{2}-1\right) \delta_{n, 0}\right\} \tau(x)=0 \quad(n=-1,0,1,2, \ldots) .
$$

On the other hand, for the case of the one-matrix models one can prove that the set of Schwinger-Dyson equations for $\tau(x)=\sqrt{Z(x)}$ is equivalent to the Virasoro constraint (2.25) with $p=2$ [5]. Since the Schwinger-Dyson equations contain necessary and sufficient information of the system, it is expected that the function $\tau(x)$ satisfying the Virasoro constraint for $p=2$ automatically becomes a $\tau$ function of the 2-reduced KP hierarchy. However, in the cases of $p \geqq 3$ the Virasoro constraint alone does not determine the system completely unless we use the fact that the $\tau(x)$ is a $\tau$ function. In these cases we thus need additional constraints on the $\tau(x)$, and it was shown in $[5,6]$ that these constraints should be the vacuum condition of the $W_{p}$ algebra:

$$
\mathbf{W}_{n}^{(k)} \tau(x)=0 \quad(k=2, \ldots, p ; n \geqq-k+1),
$$

where the $\mathbf{W}_{n}^{(k)}$ 's are the generators of the $W_{p}$ algebra constructed via quantum Miura transformation in terms of the $\mathbf{Z}_{p}$-twisted scalar fields (see Appendix C). In fact, these conditions generically determine the system up to several nonperturbative parameters, and in the particular case of the topological gravity [11], $(p, q)=(p, 1)$, they enable us to calculate all the correlation functions analytically [5].

In the following sections, we will prove that the KP hierarchy has the $W_{1+\infty}$ algebra as its fundamental symmetry under every reduction, and that the $\tau$ function satisfies the formal vacuum condition of the $W_{1+\infty}$ algebra if it obeys the constraints (2.17) and (2.20). Furthermore, we will show that they are reduced to the vacuum condition of the $W_{p}$ algebra (2.26) after we eliminate the redundant variables for the $p$-reduction.

Before going through the proof, we make a crucial remark that the string equations (2.17) and (2.20) for the $(p, q)$ quantum gravity are expressed in terms of local fermion bilinear operators. In fact, the reduction condition (2.16) is stated in terms of a local fermion bilinear operator, that is, the current operator $J(z)=: \psi^{\dagger}(z) \psi(z)$. Furthermore, due to Eq. (2.15) and the bosonization rule 
(2.12)-(2.14), the Virasoro operator acting on the $\tau$ function in Eq. (2.20) also turns out to be a local bilinear operator acting on $|g\rangle$ :

$$
\mathscr{L}_{n} \tau(x)=\left\langle 0\left|e^{\sum_{m} x_{m} J_{m}} L_{n}\right| g\right\rangle
$$

where

$$
\begin{aligned}
T(z) & =\sum_{n} L_{n} z^{-n-2} \\
& =\frac{1}{2}: J(z)^{2}: \\
& =\frac{1}{2}\left(: \partial \psi^{\dagger}(z) \psi(z):-: \psi^{\dagger}(z) \partial \psi(z):\right) .
\end{aligned}
$$

Remark. Rigorously speaking, the multi-fermion state $|g\rangle$ given in Eq. (2.6) is, in general, ill-defined. In fact, the $\tau$ function for the topological gravity ((2.1) quantum gravity) has the form [5]

$$
\tau\left(x_{1}, x_{3}, 0,0, \ldots\right)=\text { const } x_{3}^{-1 / 24} \exp \left[-\frac{x_{1}^{3}}{18 x_{3}}\right],
$$

so that we can not set $x=0$. However, since we can expand it around a generic background source $x=x^{0}$, the state $|g\rangle$ becomes well-defined if we define the $\tau$ function by

$$
\tau(x)=\left\langle 0\left|e^{\sum_{n \geq 1}\left(x_{n}+x_{n}^{0}\right) J_{n}}\right| g\right\rangle .
$$

Furthermore, if we introduce $J_{n}^{\prime}$ by

$$
\begin{aligned}
J_{n}^{\prime} & \equiv e^{-\sum_{m} x_{m}^{0} J_{m}} J_{n} e^{\sum_{m} x_{m}^{0} J_{m}} \\
& = \begin{cases}J_{n} & (n>0) \\
J_{n}+n x_{-n}^{0} & (n<0)\end{cases}
\end{aligned}
$$

then we have

$$
\langle 0| e^{\sum_{n \geqq 1}^{\sum}\left(x_{n}+x_{n}^{0}\right) J_{n}} J_{m}^{\prime}=\left\{\begin{array}{ll}
\partial_{m}\langle 0| e^{\sum_{n}\left(x_{n}+x_{n}^{0}\right) J_{n}} & (m>0) \\
|m| x_{|m|}\langle 0| e^{\sum_{n ! 1}\left(x_{n}+x_{n}^{0}\right) J_{n}} & (m<0)
\end{array},\right.
$$

which allows us to still regard $\langle 0| \exp \left(\sum_{n \geqq 1}\left(x_{n}+x_{n}^{0}\right) J_{n}\right)$ as the bosonic coherent state. Although we will formally set $x^{0}=0$ hereafter, the generalization to the cases $x^{0} \neq 0$ is easily carried out by replacing the operator $O$ which acts on the state $|g\rangle$ by

$$
O^{\prime} \equiv e^{-\sum_{m} x_{m}^{0} J_{m}} O e^{\sum_{m} x_{m}^{0} J_{m}}
$$

For example, as for the Virasoro generators in Eq. (2.27), it holds that

$$
\mathscr{L}_{n} \tau(x)=\left\langle 0\left|e^{\frac{\Sigma\left(x_{m}+x_{m}^{0}\right) J_{m}}{m}} L_{n}^{\prime}\right| g\right\rangle
$$


for

$$
L_{n}^{\prime}=e^{-\sum_{m} x_{m}^{0} J_{m}} L_{n} e^{\sum_{m} x_{m}^{0} J_{m}}
$$

\section{String Equations in Terms of UGM}

As we have seen in the previous section, fermion bilinear operators such as the $J_{n}$ 's and the $L_{n}$ 's play essential roles in the two-dimensional gravity. We call operators $W(z)$ local fermion bilinear of spin $k$ if they have the following form:

$$
W(z)=\sum_{j=0}^{k-1} c_{j}: \partial^{j} \psi^{\dagger}(z) \partial^{k-1-j} \psi(z):
$$

where $c_{j}$ are complex numbers. In particular, $J(z)$ and $T(z)$ are local fermion bilinear operators of spin 1 and 2, respectively:

$$
\begin{aligned}
& J(z)=\sum_{n \in \mathbf{Z}} J_{n} z^{-n-1}=: \psi^{\dagger}(z) \psi(z): \\
& T(z)=\sum_{n \in \mathbf{Z}} L_{n} z^{-n-2}=\frac{1}{2}\left(: \partial \psi^{\dagger}(z) \psi(z):-: \psi^{\dagger}(z) \partial \psi(z):\right) .
\end{aligned}
$$

We also call $W_{n}$ local fermion bilinear of spin $k$ if it is the $n^{\text {th }}$ mode of such an operator:

$$
W(z)=\sum_{n} W_{n} z^{-n-k}
$$

In this section, we investigate how such operators act on a decomposable state $|g\rangle$ and rewrite the string equations in terms of the UGM.

First we consider fermion bilinear operators of the general form:

$$
O=\sum_{r, s}: \psi_{r}^{\dagger} O_{r s} \psi_{s}:
$$

The crucial point is that bilinear operators can be regarded as one-body operators in terms of the first quantization. In fact, one can construct the one-body operator $o$ that corresponds to a fermion bilinear operator $O$ by taking a commutator with the fermion operators $\psi_{r}$ :

$$
\left[\psi_{r}, O\right]=\sum_{s} O_{r s} \psi_{s}
$$

which means that the one-body operator $o$ maps a one-body wave function $f(z)$

$$
=\sum_{r} a_{r} z^{-r-1 / 2} \in H \text { to }
$$

$$
o f(z) \equiv \sum_{r}\left(\sum_{s} O_{r s} s_{s}\right) z^{-r-1 / 2} .
$$

Conversely, if a one-body operator $o$ is given, we can construct a fermion bilinear operator $O$ uniquely up to an ambiguity of additive constant caused by the operator ordering. Furthermore, if fermion bilinear operators $\mathrm{O}_{1}$ and $\mathrm{O}_{2}$ correspond to one-body operators $o_{1}$ and $o_{2}$, respectively, then the commutator of $O_{1}$ and $O_{2}$ corresponds to the commutator of $o_{1}$ and $o_{2}$ :

$$
O_{1} \leftrightarrow o_{1}, \quad O_{2} \leftrightarrow o_{2} \Rightarrow\left[O_{1}, O_{2}\right] \leftrightarrow\left[o_{1}, o_{2}\right]
$$


Thus we have

Lemma 3.1. If a set of one-body operator forms a Lie algebra $w$, then the set of corresponding fermion bilinear operators forms a central extension of $w$.

Furthermore, for any value of $\varepsilon$ the action of $\exp (\varepsilon O)$ on a decomposable multifermion state $|g\rangle$ is represented over the UGM as

$$
e^{\varepsilon o}|g\rangle \leftrightarrow e^{\varepsilon o} V_{g}=\left[e^{\varepsilon o} \xi^{(0)}(z), e^{\varepsilon o} \xi^{(1)}(z), \ldots\right],
$$

where $V_{g}=\left[\xi^{(0)}(z), \xi^{(1)}(z), \ldots\right]$ is the subspace of $H$ corresponding to $|g\rangle$. From this fact, it is obvious that the following lemma holds:

\section{Lemma 3.2.}

$$
O|g\rangle=\text { const }|g\rangle \Leftrightarrow o V_{g} \subset V_{g}
$$

This lemma will be frequently used below.

If we restrict ourselves to the local fermion bilinear operators, the corresponding one-body operators become local differential operators with respect to $z$. In fact, for an operator having the form

$$
\begin{gathered}
W_{n}=\oint \frac{d w}{2 \pi i} w^{n+k-1} W(w), \\
W(w)=: \partial^{j} \psi^{\dagger}(w) \partial^{k-1-j} \psi(w):,
\end{gathered}
$$

the commutator with $\psi(z)$ gives

$$
\begin{aligned}
{\left[\psi(z), W_{n}\right] } & =-\oint_{z} \frac{d w}{2 \pi i} w^{n+k-1} \psi(z): \partial^{j} \psi^{\dagger}(w) \partial^{k-1-j} \psi(w): \\
& =(-1)^{j}\left(\frac{d}{d z}\right)^{j} z^{n+k-1}\left(\frac{d}{d z}\right)^{k-1-j} \psi(z)
\end{aligned}
$$

This equation indicates that the corresponding one-body operator is given by 4

$$
\begin{aligned}
w_{n} & =(-1)^{j}\left(\frac{d}{d z}\right)^{j} z^{n+k-1}\left(\frac{d}{d z}\right)^{k-1-j} \\
& =(-1)^{j} \sum_{l=0}^{j}\left(\begin{array}{l}
j \\
l
\end{array}\right)[n+k-1]_{l} z^{n+k-1-l}\left(\frac{d}{d z}\right)^{k-1-l},
\end{aligned}
$$

where $[m]_{n} \equiv m ! /(m-n)$ !. In particular, the one-body operators corresponding to the current $J_{n}$ and the energy-momentum tensor $L_{n}$ are as follows:

$$
\begin{aligned}
& J_{n} \leftrightarrow w_{n}^{(1)}=z^{n}, \\
& L_{n} \leftrightarrow w_{n}^{(2)}=-\left[z^{n+1} \frac{d}{d z}+\frac{n+1}{2} z^{n}\right] .
\end{aligned}
$$

If we take into account all modes of all the local fermion bilinear operators, they generate a Lie algebra called the $W_{1+\infty}$ algebra. From Eq. (3.12) it is easily seen

\footnotetext{
${ }^{4}$ If we define the $\tau$ function as (2.29), then this expression for $w_{n}$ must be replaced by $w_{n}^{\prime}$ $=\exp \left(-\sum_{m} x_{m}^{0} z^{m}\right) w_{n} \exp \left(\sum_{m} x_{m}^{0} z^{m}\right)$
} 
that the corresponding one-body operators form a Lie algebra of differential operators that is spanned by

$$
z^{n}\left(\frac{d}{d z}\right)^{l} \quad\left(n \in \mathbf{Z}, l \in \mathbf{Z}_{+}=\{0,1,2, \ldots\}\right),
$$

which we call the $w_{1+\infty}$ algebra. Obviously the $W_{1+\infty}$ algebra is a central extension of the $w_{1+\infty}$ algebra. In fact, the central charge $c$ of the Virasoro subalgebra of $W_{1+\infty}$ is 1 , whereas that of $w_{1+\infty}$ is 0 .

Using these one-body operators, we can express the string Eqs. (2.17) and (2.20) for the $(p, q)$ quantum gravity in terms of the UGM. As is shown in the next section, these two equations are equivalent to the following weaker conditions on the $\tau$ function:

$$
\begin{aligned}
\partial_{p} \tau(x) & =\text { const } \tau(x), \\
\mathscr{L}_{-p} \tau(x) & =\text { const } \tau(x),
\end{aligned}
$$

or equivalently,

$$
\begin{gathered}
J_{p}|g\rangle=\text { const }|g\rangle, \\
L_{-p}|g\rangle=\text { const }|g\rangle .
\end{gathered}
$$

Then Lemma 3.2 together with the relations in (3.13) tells that these equations are equivalent to

$$
\begin{gathered}
w_{p}^{(1)} V_{g}=z^{p} V_{g} \subset V_{g}, \\
w_{-p}^{(2)} V_{g}=-\left[z^{-p+1} \frac{d}{d z}+\frac{-p+1}{2} z^{-p}\right] V_{g} \subset V_{g} .
\end{gathered}
$$

Therefore the subspace $V_{g}$ is invariant under any differential operator that is constructed by repeatedly taking products and linear combinations of $w_{p}^{(1)}$ and $w_{-p}^{(2)}$. In other words, if we define $r^{+}(p)$ as the associative subalgebra of differential operators that is generated by $w_{p}^{(1)}$ and $w_{-p}^{(2)}$, we have

$$
o V_{g} \subset V_{g} \text { for }{ }^{\forall} o \in r^{+}(p) \text {. }
$$

Since the commutator of $w_{p}^{(1)}$ and $w_{-p}^{(2)}$ is a $c$-number

$$
\left[w_{p}^{(1)}, \frac{1}{p} w_{-p}^{(2)}\right]=1
$$

and the differential operators $\left(w_{p}^{(1)}\right)^{n}\left(w_{-p}^{(2)}\right)^{l}(n, l=0,1,2, \ldots)$ are all linearly independent to each other, any element of $r^{+}(p)$ is uniquely expressed as

$$
\sum_{n, l=0}^{\infty} c_{n l}\left(w_{p}^{(1)}\right)^{n}\left(w_{-p}^{(2)}\right)^{l}
$$

where the $c_{n l}$ 's are complex numbers. Thus we have the following theorem:

Theorem 3.3. Let $\tau(x)$ be a $\tau$ function of the KP hierarchy that satisfies the string equations of $(p, q)$ quantum gravity (3.15) and (3.16). Then the corresponding element $V_{g}$ of the UGM satisfies

$$
r^{+}(p) V_{g}=V_{g}
$$

where $r^{+}(p)=\left\{\sum_{n, l=0}^{\infty} c_{n l}\left(w_{p}^{(1)}\right)^{n}\left(w_{-p}^{(2)}\right)^{l}, c_{n l} \in \mathbf{C}\right\}$. 
As is shown in the next section, we can reinterpret Eq. (3.22) in terms of the $\tau$ function by using Lemma 3.2 again. There we will see that the structure of the $W_{1+\infty}$ algebra arises in a natural way. Here, before going back to the $\tau$ function, we analyze the Lie algebraic structures of the set $r^{+}(p)$ of one-body operators that appears in Eq. (3.22).

First we introduce the following notations for $p \geqq 2$ :

$$
\begin{gathered}
r=w_{1+\infty}=\left\{\sum_{n \in \mathbf{Z}} \sum_{l \in \mathbf{Z}_{+}} c_{n l} z^{n}\left(-\frac{d}{d z}\right)^{l}\right\}, \\
r^{+}=w_{1+\infty}^{+}=\left\{\sum_{n \in \mathbf{Z}_{+}} \sum_{l \in \mathbf{Z}_{+}} c_{n l} z^{n}\left(-\frac{d}{d z}\right)^{l}\right\}, \\
r(p)=w_{1+\infty}(p)=\left\{\sum_{n \in \mathbf{Z}} \sum_{l \in \mathbf{Z}_{+}} c_{n l}\left(w_{p}^{(1)}\right)^{n}\left(\frac{1}{p} w_{-p}^{(2)}\right)^{l}\right\}, \\
r^{+}(p)=w_{1+\infty}^{+}(p)=\left\{\sum_{n \in \mathbf{Z}_{+}} \sum_{l \in \mathbf{Z}_{+}} c_{n l}\left(w_{p}^{(1)}\right)^{n}\left(\frac{1}{p} w_{-p}^{(2)}\right)^{l}\right\} .
\end{gathered}
$$

Here, $r, r^{+}, r(p)$, and $r^{+}(p)$ are, as a set, identical to $w_{1+\infty}, w_{1+\infty}^{+}, w_{1+\infty}(p)$, and $w_{1+\infty}^{+}(p)$, respectively. We introduce, however, different symbols for them in order to indicate whether we regard them as associative algebras or Lie algebras. In other words, when we call them $r$, we consider not only commutators but also products as differential operators. ${ }^{5}$ In addition to the trivial relations

$$
\begin{array}{cc}
r^{+} \subset r, \quad r^{+}(p) \subset r(p) \subset r \quad \text { (as associative subalgebras), } \\
w_{1+\infty}^{+} \subset w_{1+\infty}, \quad w_{1+\infty}^{+}(p) \subset w_{1+\infty}(p) \subset w_{1+\infty} \quad \text { (as Lie subalgebras), }
\end{array}
$$

we have the following isomorphisms, which indicate that the $w_{1+\infty}$ algebra has an infinite-fold self-similar structure:

\section{Lemma 3.4.}

$$
\begin{gathered}
r(p) \cong r, \quad r^{+}(p) \cong r^{+} \quad \text { (as associative algebras) }, \\
w_{1+\infty}(p) \cong w_{1+\infty}, \quad w_{1+\infty}^{+}(p) \cong w_{1+\infty}^{+} \quad \text { (as Lie algebras). }
\end{gathered}
$$

Proof. As is clear from the definition, $r$ is generated by $w_{n}^{(1)}=z^{n}(n \in \mathbf{Z})$ and $w_{-1}^{(2)}$ $=-d / d z$, and the structure of $r$ is completely specified by the following relations among them:

$$
\begin{gathered}
w_{n}^{(1)}=\left(w_{1}^{(1)}\right)^{n}, \\
{\left[w_{1}^{(1)}, w_{-1}^{(2)}\right]=1 .}
\end{gathered}
$$

On the other hand, $r(p)$ is generated by $w_{n p}^{(1)}=z^{n p}(n \in \mathbf{Z})$ and $w_{-p}^{(2)}$ with the relations

$$
\begin{gathered}
w_{n p}^{(1)}=\left(w_{p}^{(1)}\right)^{n}, \\
{\left[w_{p}^{(1)}, \frac{1}{p} w_{-p}^{(2)}\right]=1 .}
\end{gathered}
$$

5 Infact, theso-called lone-star product of the $W_{1+\infty}$ algebra [7] is nothing but the usual product in $r$, when wetranslate the set of fermion bilinear operators, $W_{1+\infty}$, into the set of corresponding one-body operators, $w_{1+\infty}$ 
Therefore $r(p)$ is isomorphic to $r$ as an associative algebra through the following mapping:

$$
\begin{gathered}
\bar{w}_{1}^{(1)} \equiv w_{p}^{(1)} \mapsto w_{1}^{(1)}, \\
\bar{w}_{-1}^{(2)} \equiv \frac{1}{p} w_{-p}^{(2)} \mapsto w_{-1}^{(2)} .
\end{gathered}
$$

Furthermore, since $r^{+}(p)$ is generated by $w_{p}^{(1)}$ and $w_{-p}^{(2)}$, and $r^{+}$is generated by $w_{1}^{(1)}$ and $w_{-1}^{(2)}$, these two associative algebras are isomorphic under the above mapping.

To express this isomorphism more explicitly, we note the following relation:

$$
\begin{gathered}
\bar{w}_{-1}^{(2)}=\frac{1}{p} w_{-p}^{(2)}=-\frac{1}{p}\left(z^{-p+1} \frac{d}{d z}+\frac{-p+1}{2} z^{-p}\right)=z^{(p-1) / 2}\left(-\frac{d}{d \lambda}\right) z^{-(p-1) / 2}, \\
\bar{w}_{1}^{(1)}=w_{p}^{(1)}=z^{p}=z^{(p-1) / 2}(\lambda) z^{-(p-1) / 2}
\end{gathered}
$$

where $\lambda=z^{p}$. Then we see that the $w_{1+\infty}(p)$ algebra and the $w_{1+\infty}$ algebra are related via

$$
w_{1+\infty}(p)=z^{(p-1) / 2}\left(\left.w_{1+\infty}\right|_{\text {with } z \text { replaced by } \lambda) z^{-(p-1) / 2}}\right. \text {. }
$$

In order to see how $w_{1+\infty}(p)$ is imbedded in $w_{1+\infty}$ more explicitly, we introduce the following basis of the $w_{1+\infty}$ algebra:

$$
w_{n}^{(k)}=(-1)^{k-1} \sum_{l=0}^{k-1} \frac{1}{l !} \frac{\left([k-1]_{l}\right)^{2}}{[2 k-2]_{l}}[n+k-1]_{l} z^{n+k-1-l}\left(\frac{d}{d z}\right)^{k-1-l},
$$

which corresponds to the standard basis of the $W_{1+\infty}$ algebra ${ }^{6}[7]$ :

$$
W^{(k)}(z)=\sum_{n} W_{n}^{(k)} z^{-n-k}=\frac{(k-1) !}{2^{k-1}(2 k-3) ! !} \sum_{j=0}^{k-1}(-1)^{j}\left(\begin{array}{c}
k-1 \\
j
\end{array}\right)^{2}: \partial^{k-1-j} \psi^{\dagger}(z) \partial^{j} \psi(z):
$$

Then by using Eq. (3.36) the corresponding basis of $w_{1+\infty}(p)$ is expressed as

$$
\bar{w}_{n}^{(k)}=(-1)^{k-1} \sum_{l=0}^{k-1} \frac{1}{l !} \frac{\left([k-1]_{l}\right)^{2}}{[2 k-2]_{l}}[n+k-1]_{l} z^{(p-1) / 2} \lambda^{n+k-1-l}\left(\frac{d}{d \lambda}\right)^{k-1-l} z^{-(p-1) / 2} .
$$

The right-hand side of this equation can be expressed as a linear combination of the $w_{n}^{(k)}$ 's. For example,

$$
\begin{gathered}
\bar{w}_{n}^{(1)}=w_{n p}^{(1)}, \\
\bar{w}_{n}^{(2)}=\frac{1}{p} w_{n p}^{(2)}, \\
\bar{w}_{n}^{(3)}=\frac{1}{p^{2}}\left\{w_{n p}^{(3)}+\frac{1}{12}\left(p^{2}-1\right) w_{n p}^{(1)}\right\}, \\
\bar{w}_{n}^{(4)}=\frac{1}{p^{3}}\left\{w_{n p}^{(4)}+\frac{7}{20}\left(p^{2}-1\right) w_{n p}^{(2)}\right\} .
\end{gathered}
$$

6 Here, $(-1) ! ! \equiv 1$ 
As a final remark in this section, we point out the following properties of $w_{1+\infty}^{+}$ and $w_{1+\infty}^{+}(p)$, which we use in the next section:

Lemma 3.5. Any element of $w_{1+\infty}^{+}$can be expressed as a commutator of two elements of $w_{1+\infty}^{+}$. The same statement holds for $w_{1+\infty}^{+}(p)$.

Proof. For any element of $w_{1+\infty}^{+}$, we have

$$
\sum_{k, l \in \mathbf{Z}_{+}} c_{k, l} z^{k}\left(\frac{d}{d z}\right)^{l}=\left[\frac{d}{d z}, \sum_{k, l \in \mathbf{Z}_{+}} \frac{c_{k, l}}{k+1} z^{k+1}\left(\frac{d}{d z}\right)^{l}\right],
$$

which proves the assertion for $w_{1+\infty}^{+}$. The same assertion holds for $w_{1+\infty}^{+}(p)$ because it is isomorphic to $w_{1+\infty}^{+}$.

\section{4. $W_{1+\infty}$ Constraint}

In the preceding sections we have investigated the structure of the string equation in terms of the UGM. In this section we will rewrite Eq. (3.22) as a set of differential equations for $\tau(x)$.

As we have seen in the previous section, if a set of one-body operators is given, we can construct a corresponding set of fermion bilinear operators up to the operator ordering ambiguities. One way to fix the ambiguities is to introduce the usual normal ordering for the fermion operators (2.8). In fact, the $W_{1+\infty}$ algebra can be regarded as the set of fermion bilinear operators constructed from $w_{1+\infty}$ through this normal ordering. Similarly, we define $W_{1+\infty}^{+}$as the subset of $W_{1+\infty}$ that is constructed from $w_{1+\infty}^{+}$by using the same normal ordering. As is clear from Eq. (3.12), $W_{1+\infty}^{+}$is spanned by $W_{n}^{(k)}$ 's satisfying $n \geqq-k+1$. A crucial property of the $W_{1+\infty}^{+}$is the following:

Lemma 4.1. $W_{1+\infty}^{+}$forms a Lie algebra without a central term. In other words, $W_{1+\infty}^{+}$closes under the commutator.

Proof. Since $w_{1+\infty}^{+}$is a Lie algebra, it is obvious that $W_{1+\infty}^{+}$is a Lie algebra with possible central terms. In order to show that the central terms vanish, we consider the $c$-number term of the operator product expansion between fermion bilinear operators $W^{(k)}(z)$ and $W^{(l)}(w)$ of spin $k$ and $l$, respectively:

$$
W^{(k)}(z) W^{(l)}(w)=\text { const } \frac{1}{(z-w)^{k+l}}+(\text { fermion bilinear operators }),
$$

which means that the $c$-number term of the commutator $\left[W_{n}^{(k)}, W_{m}^{(l)}\right]$ is given by

$$
\text { const }[n+k-1]_{k+l-1} \delta_{n+m, 0}=\operatorname{const}(n+k-1) \ldots(n-l+1) \delta_{n+m, 0} .
$$

Then it is easy to see that one of the factors in Eq. (4.2) vanishes if $n \geqq-k+1$ and $m \geqq-l+1$. Thus we find that $W_{1+\infty}^{+}$closes under the commutator.

We then define $W_{1+\infty}(p)$ and $W_{1+\infty}^{+}(p)$ as the sets of fermion bilinear operators that are constructed from $w_{1+\infty}(p)$ and $w_{1+\infty}^{+}(p)$, respectively. Here again we have the operator ordering ambiguities for the $0^{\text {th }}$ modes of the fermion bilinear operators. We can, however, show the following:

Lemma 4.2. There exists such a proper definition of the $0^{\text {th }}$ modes that $W_{1+\infty}^{+}(p)$ closes under the commutator. Furthermore, any element of $W_{1+\infty}^{+}(p)$ can be expressed as a commutator of two elements of $W_{1+\infty}^{+}(p)$. 
Proof. The isomorphisms (3.36) between $w_{1+\infty}$ and $w_{1+\infty}(p)$ can be regarded as a conformal mapping from $z$ to $\lambda=z^{p}$ :

$$
\psi^{\prime}(\lambda)=\left(\frac{d \lambda}{d z}\right)^{-1 / 2} \psi(z) .
$$

Therefore, if we fix the operator ordering by introducing the new normal ordering as the subtraction of the singular part in the ( $p$-sheeted) $\lambda$ plane, such as

$$
\circ \psi^{\prime \dagger}\left(\lambda^{\prime}\right) \psi^{\prime}(\lambda) \stackrel{\circ}{\circ} \psi^{\prime \dagger}\left(\lambda^{\prime}\right) \psi^{\prime}(\lambda)-\frac{1}{\lambda^{\prime}-\lambda},
$$

then the structure of the $c$-number terms of the operator product expansions for $W_{1+\infty}(p)$ in the $\lambda$ plane is exactly the same as that of $W_{1+\infty}^{+}$in the $z$ plane. Thus the same argument as in Lemma 3.5 leads to the closedness of $W_{1+\infty}^{+}(p)$ under the commutator. The latter assertion follows immediately from this fact and Lemma 3.5.

As is well known, the difference between the $\lambda$ plane normal ordering and the usual normal ordering can be calculated as the Schwarzian terms associated with the transformation $z \mapsto \lambda=z^{p}$. For example, the generators of the $W_{1+\infty}(p)$ corresponding to (3.40)-(3.43) take the following forms:

$$
\begin{gathered}
\bar{W}_{n}^{(1)}=W_{n p}^{(1)}, \\
\bar{W}_{n}^{(2)}=\frac{1}{p}\left\{W_{n p}^{(2)}+\frac{1}{24}\left(p^{2}-1\right) \delta_{n, 0}\right\}, \\
\bar{W}_{n}^{(3)}=\frac{1}{p^{2}}\left\{W_{n p}^{(3)}+\frac{1}{12}\left(p^{2}-1\right) W_{n p}^{(1)}\right\}, \\
\bar{W}_{n}^{(4)}=\frac{1}{p^{3}}\left\{W_{n p}^{(4)}+\frac{7}{20}\left(p^{2}-1\right) W_{n p}^{(2)}+\frac{7}{960}\left(p^{2}-1\right)^{2} \delta_{n, 0}\right\} .
\end{gathered}
$$

Note the appearance of the additional $c$-number correction terms compared to (3.40)-(3.43). It is obvious from the construction that they satisfy the commutation relations of the $W_{1+\infty}$ algebra with central charge $c=p$.

After the rather lengthy preparation given above, we can finally prove that a $\tau$ function of the $p$-reduced KP hierarchy satisfies the vacuum condition of the $W_{1+\infty}(p)$ algebra when it obeys the string Eq. (2.20). Here, we can start with weaker assumptions

$$
\begin{gathered}
\partial_{p} \tau(x)=\text { const } \tau(x), \\
\mathscr{L}_{-p} \tau(x)=\text { const } \tau(x),
\end{gathered}
$$

or equivalently

$$
\begin{gathered}
J_{p}|g\rangle=\text { const }|g\rangle, \\
L_{-p}|g\rangle=\text { const }|g\rangle .
\end{gathered}
$$

In Theorem 3.3, we found that these equations are equivalent to

$$
r^{+}(p) V_{g}=w_{1+\infty}^{+}(p) V_{g}=V_{g} \text {. }
$$


By using Lemma 3.2 again, this means that $|g\rangle$ is a simultaneous eigenstate of $W_{1+\infty}^{+}(p)$ :

$$
O|g\rangle=\text { const }|g\rangle \text { for }{ }^{\forall} O \in W_{1+\infty}^{+}(p) .
$$

Furthermore, Lemma 4.2 asserts that all of these constants vanish. In fact, any element $O$ of $W_{1+\infty}^{+}(p)$ can be expressed as

$$
O=\left[O_{1}, O_{2}\right], \quad O_{1}, O_{2} \in W_{1+\infty}^{+}(p) .
$$

Therefore, we have $O|g\rangle=O_{1} O_{2}|g\rangle-O_{2} O_{1}|g\rangle=0$, because $|g\rangle$ is a simultaneous eigenstate of $W_{1+\infty}^{+}(p)$.

We thus have proved the following theorem.

Theorem 4.3. Let $\tau(x)$ be a $\tau$ function of the KP hierarchy that satisfies the conditions (4.9) and (4.10). Then the decomposable fermion state $|g\rangle$ corresponding to the $\tau$ function satisfies

$$
O|g\rangle=0 \quad \text { for } \quad{ }^{\forall} O \in W_{1+\infty}^{+}(p),
$$

that is,

$$
\bar{W}_{n}^{(k)}|g\rangle=0 \quad(k=1,2, \ldots ; n \geqq-k+1) .
$$

This theorem can be restated in terms of bosons as follows. By using the bosonization rule (2.12)-(2.14), the normal ordering of two fermion operators

$$
: \partial^{k} \psi^{\dagger}(w) \partial^{l} \psi(z)
$$

is expanded in powers of $w-z$ as

$$
\begin{aligned}
& =\partial_{w}^{k} \partial_{z}^{l}\left(\frac{1}{w-z}: e^{\phi(w)-\phi(z)}:-\frac{1}{w-z}\right) \\
& =\sum_{j=1}^{\infty} \sum_{r=0}^{j-1-k}(-1)^{r}\left(\begin{array}{l}
l \\
r
\end{array}\right) \frac{1}{j \cdot(j-1-k-r) !}(w-z)^{j-1-k-r} \partial^{l-r} P^{(j)}(z),
\end{aligned}
$$

where $P^{(j)}(z)$ is defined by

$$
P^{(j)}(z) \equiv: e^{-\phi(z)} \partial_{z}^{j} e^{\phi(z)}:
$$

and $::$ stands for the normal ordering for bosonic operators. Considering the coefficient of $(w-z)^{0}$ in Eq. (4.19), we have

$$
: \partial^{k} \psi^{\dagger}(z) \partial^{l} \psi(z):=\sum_{j=k+1}^{k+l+1} \frac{1}{j}(-1)^{j-1-k}\left(\begin{array}{c}
l \\
j-1-k
\end{array}\right) \partial^{k+l-j+1} P^{(j)}(z)
$$

Thus, by substituting this expression into Eq. (3.38), we obtain the bosonic realization of the $W_{1+\infty}$ algebra:

$$
W^{(k)}(z)=\sum_{l=0}^{k-1} \frac{(-1)^{l}}{(k-l) \cdot l !} \frac{\left([k-1]_{l}\right)^{2}}{[2 k-2]_{l}} \partial^{l} P^{(k-l)}(z),
$$

the first few of which are

$$
\begin{gathered}
W^{(1)}(z)=J(z), \\
W^{(2)}(z)=\frac{1}{2}: J(z)^{2}:, \\
W^{(3)}(z)=\frac{1}{3}: J(z)^{3}:, \\
W^{(4)}(z)=\frac{1}{4}\left[: J(z)^{4}:+\frac{2}{5}: J(z) \partial^{2} J(z):-\frac{3}{5}:(\partial J(z))^{2}:\right] .
\end{gathered}
$$


Then by using the equations such as (4.5)-(4.8), the generators of $W_{1+\infty}(p)$ are expressed as follows:

$$
\begin{gathered}
\bar{W}_{n}^{(1)}=J_{n p}, \\
p \bar{W}_{n}^{(2)}=\frac{1}{2} \sum_{a+b=n p}: J_{a} J_{b}:+\frac{1}{24}\left(p^{2}-1\right) \delta_{n, 0}, \\
p^{2} \bar{W}_{n}^{(3)}=\frac{1}{3} \sum_{a+b+c=n p}: J_{a} J_{b} J_{c}:+\frac{1}{12}\left(p^{2}-1\right) J_{n p}, \\
p^{3} \bar{W}_{n}^{(4)}=\frac{1}{4} \sum_{a+b+c+d=n p}: J_{a} J_{b} J_{c} J_{d}:-\frac{1}{4} \sum_{a+b=n p}(a+1)(b+1): J_{a} J_{b}: \\
+\frac{1}{20}(n p+2)(n p+3) \sum_{a+b=n p}: J_{a} J_{b}:+\frac{7}{40}\left(p^{2}-1\right) \sum_{a+b=n p}: J_{a} J_{b}: \\
+\frac{7}{960}\left(p^{2}-1\right)^{2} \delta_{n, 0},
\end{gathered}
$$

Hence, Eq. (4.17) is rewritten as a set of differential equations for the $\tau$ function:

$$
\overline{\mathscr{W}}_{n}^{(k)} \tau(x)=0 \quad(k=1,2,3, \ldots ; n \geqq-k+1),
$$

where the differential operator $\bar{W}_{n}^{(k)}$ is obtained by replacing all the $J_{n}$ 's in the $\bar{W}_{n}^{(k)}$ by

$$
\mathscr{J}_{n} \equiv\left\{\begin{array}{ll}
\partial_{n} & (n>0) \\
|n| x_{|n|} & (n<0)
\end{array}\right. \text {. }
$$

Note that the first equation $\overline{\mathscr{W}}_{n}^{(1)} \tau(x)=\frac{\partial}{\partial x_{n p}} \tau(x)=0$ shows that the $\tau(x)$ is a $\tau$ function of the $p$-reduced KP hierarchy. As was discussed in [5] it is expected that a function $\tau(x)$ satisfying Eq. (4.31) automatically becomes a $\tau$ function of the KP hierarchy, since Eq. (4.31) determines the function $\tau(x)$ completely at least for the case of topological fields [5].

\section{5. $W_{p}$ Constraint}

We have shown that a $\tau$ function of the KP hierarchy under the conditions (4.9) and (4.10) is a $\tau$ function of the $p$-reduced KP hierarchy which satisfies the vacuum condition of the $W_{1+\infty}(p)$. However, in the expressions (4.30)-(4.32) there appear redundant variables for the $p$-reduced KP hierarchy, that is, $J_{n p}(n \in \mathbf{Z})$. In this section we show that after the elimination of these redundant variables the $W_{1+\infty}$ algebra with central charge $c=p$ is reduced to the $W_{p}$ algebra with $c=p-1$.

As we have seen in the previous section, the generators of $W_{1+\infty}(p)$ have simple forms when they are expressed on the $p$-sheeted $\lambda$ plane which is the image space of the conformal transformation $z \mapsto \lambda=z^{p}$. More explicitly, we first define the operators $W_{n}^{(k)}(\lambda)$ on the $p$-sheeted $\lambda$ plane by Eq. (4.22) with $z$ replaced by $\lambda$ :

$$
\begin{gathered}
W^{(k)}(\lambda)=\sum_{l=0}^{k-1} \frac{(-1)^{l}}{(k-l) \cdot l !} \frac{\left([k-1]_{l}\right)^{2}}{[2 k-2]_{l}} \partial_{\lambda}^{l} P^{(k-l)}(\lambda), \\
P^{(j)}(\lambda)={ }_{\circ}^{\circ} e^{-\phi(\lambda)} \partial_{\lambda}^{j} e^{\phi(\lambda) \circ}{ }_{\circ}{ }_{\circ}\left(\partial_{\lambda}+J(\lambda)\right)^{j \circ} 1 .
\end{gathered}
$$


Here, $\partial_{\lambda} \phi(\lambda)=J(\lambda)=\frac{1}{p} \sum_{n} \lambda^{-n / p-1} J_{n}$, and $: \circ$ stands for the "minimal" normal ordering on the $p$-sheeted $\lambda$ plane, by which we mean the following procedure:

$$
\begin{gathered}
\stackrel{\circ}{\circ}(\lambda)^{\circ}=J(\lambda), \\
: J(\lambda)^{3 \circ}=\lim _{\substack{\lambda^{\prime \prime} \rightarrow \lambda \\
\lambda^{\prime} \rightarrow \lambda}}\left\{J\left(\lambda^{\prime \prime}\right) J\left(\lambda^{\prime}\right) J(\lambda)\right. \\
\left.-\frac{1}{\left(\lambda^{\prime \prime}-\lambda^{\prime}\right)^{2}} J(\lambda)-\frac{1}{\left(\lambda^{\prime \prime}-\lambda\right)^{2}} J\left(\lambda^{\prime}\right)-\frac{1}{\left(\lambda^{\prime}-\lambda\right)^{2}} J\left(\lambda^{\prime \prime}\right)\right\},
\end{gathered}
$$

Since we are considering the $p$-sheeted $\lambda$ plane, each value of $\lambda$ corresponds to $p$ different points, which we denote by $\lambda_{1}, \ldots, \lambda_{p}$. Then the generators of $W_{1+\infty}(p)$ are expressed as

$$
\bar{W}^{(k)}(\lambda)=\sum_{n} \bar{W}_{n}^{(k)} \lambda^{-n-1}=W^{(k)}\left(\lambda_{1}\right)+W^{(k)}\left(\lambda_{2}\right)+\ldots+W^{(k)}\left(\lambda_{p}\right) .
$$

Note that this expression gives a single-valued function of $\lambda$ because the right-hand side is invariant under the transformation: $\lambda \mapsto \exp [2 \pi i] \lambda$, which generates a permutation of the $\lambda_{i}$ 's.

In order to investigate further the structure of the $W_{1+\infty}(p)$ constraint, we introduce the elementary symmetric polynomials of $J\left(\lambda_{1}\right), \ldots, J\left(\lambda_{p}\right)$ as

$$
S^{(k)}(\lambda)=\sum_{1 \leqq i_{1}<i_{2}<\ldots<i_{k} \leqq p} J\left(\lambda_{i_{1}}\right) \ldots J\left(\lambda_{i_{k}}\right) \quad(1 \leqq k \leqq p) .
$$

Here, the ordering of the operators on the right-hand side need not be specified because we have for two different points $\lambda$ and $\lambda^{\prime}$ on the $p$-sheeted $\lambda$ plane

$$
\left[J\left(\lambda^{\prime}\right), J\left(\lambda^{\prime \prime}\right)\right]=0 \text { if }\left|\lambda^{\prime}\right|=\left|\lambda^{\prime \prime}\right| .
$$

Furthermore it is apparent that the $S^{(k)}$ 's are single-valued functions of $\lambda$ for the same reason as in Eq. (5.6). Next we introduce another type of product for two local operators on the single-sheeted $\lambda$ plane as

$$
\left(O_{1}(\lambda), O_{2}(\lambda)\right)=\oint_{\lambda} \frac{d \lambda^{\prime}}{2 \pi i} \frac{O_{1}\left(\lambda^{\prime}\right) O_{2}(\lambda)}{\lambda^{\prime}-\lambda} .
$$

Although this product is neither commutative nor associative, it plays a crucial role in the following argument.

For any set $\mathscr{S}$ of local operators on the single-sheeted $\lambda$ plane, we can construct an algebra $R[\mathscr{S}]$ of operators by repeatedly taking $\lambda$-derivatives, linear combinations and the product (5.9). Then it is expected that the following statement holds, although we do not have a complete proof:

Lemma 5.1 (conjecture).

$$
R\left[\left\{S^{(k)}(\lambda) ; k=1,2, \ldots, p\right\}\right]=R\left[\left\{\bar{W}^{(k)}(\lambda) ; k=1,2, \ldots\right\}\right] .
$$

In other words, $\bar{W}^{(l)} \in R\left[\left\{S^{(k)}(\lambda) ; k=1,2, \ldots, p\right\}\right]$ and $S^{(l)} \in R\left[\left\{\bar{W}^{(k)}(\lambda) ; k=1,2, \ldots\right\}\right]$. 
This equation becomes obvious if we ignore all terms including $\lambda$-derivatives of $J(\lambda)$. In fact, we have

$$
\bar{W}^{(k)}(\lambda)=\frac{1}{k} \sum_{i=1}^{p} \circ J\left(\lambda_{i}\right)^{k \circ}+\text { terms including } \partial J(\lambda), \partial^{2} J(\lambda), \ldots,
$$

and the product (5.9) is equal to the normal ordering up to terms with derivatives of $J(\lambda)$, e.g.,

$$
\left(S^{(k)}(\lambda), S^{(l)}(\lambda)\right)={ }_{\circ}^{\circ} S^{(k)}(\lambda) S^{(l)}(\lambda) \circ+\text { terms including } \partial J(\lambda), \partial^{2} J(\lambda), \ldots
$$

Therefore, if we keep terms only with the form of polynomials of $J(\lambda)$, the statement of Lemma 5.1 is nothing but the fundamental theorem for symmetric polynomials. The remarkable fact is that the use of the product (5.9) gives Eq. (5.10) exactly. As is shown in Appendix B, we have checked this for operators of spin $k=1,2, \ldots, 6$. The explicit relations between the $\bar{W}^{(k)}$ 's and $S^{(k)}$ 's are given by, for example,

$$
\begin{aligned}
\bar{W}^{(1)}= & S^{(1)}, \\
2 \bar{W}^{(2)}= & \left(S^{(1)}, S^{(1)}\right)-2 S^{(2)}, \\
3 \bar{W}^{(3)}= & \left(S^{(1)},\left(S^{(1)}, S^{(1)}\right)\right)-3\left(S^{(1)}, S^{(2)}\right)+3 S^{(3)}, \\
4 \bar{W}^{(4)}+ & \frac{3}{5} \partial^{2} \bar{W}^{(2)} \\
= & \left\{\frac{1}{2}\left(S^{(1)},\left(S^{(1)},\left(S^{(1)}, S^{(1)}\right)\right)\right)+\frac{1}{2}\left(\left(S^{(1)}, S^{(1)}\right),\left(S^{(1)}, S^{(1)}\right)\right)\right\} \\
& -\left\{2\left(S^{(1)},\left(S^{(1)}, S^{(2)}\right)\right)+\left(\left(S^{(1)}, S^{(1)}\right), S^{(2)}\right)+\left(S^{(2)},\left(S^{(1)}, S^{(1)}\right)\right)\right\} \\
& +4\left(S^{(1)}, S^{(3)}\right)+2\left(S^{(2)}, S^{(2)}\right)-4 S^{(4)} .
\end{aligned}
$$

Assuming the correctness of Lemma 5.1, we have

Theorem 5.2. The vacuum condition of the $W_{1+\infty}$ algebra

$$
\bar{W}_{n}^{(k)}|g\rangle=0 \quad(k=1,2,3, \ldots ; n \geqq-k+1)
$$

is equivalent to

$$
S_{n}^{(k)}|g\rangle=0 \quad(k=1,2,3, \ldots, p ; n \geqq-k+1),
$$

where $S^{(k)}(\lambda)=\sum_{n} \lambda^{-n-k} S_{n}^{(k)}$.

Proof. We introduce such an abbreviation as $U^{(k)}|g\rangle=0$, if a state $|g\rangle$ is annihilated by the modes of spin $k$ operator $U^{(k)}(\lambda)$ with $n \geqq-k+1$, that is,

$$
U_{n}^{(k)}|g\rangle=0 \quad(\text { for } n \geqq-k+1),
$$

where $U^{(k)}=\sum_{n} \lambda^{-n-k} U_{n}^{(k)}$. Obviously, we have

$$
\begin{aligned}
& U^{(k)}|g\rangle=0 \Rightarrow\left(\partial^{l} U^{(k)}\right)|g\rangle=0, \\
& U^{(k)}|g\rangle=0, \quad V^{(k)}|g\rangle=0 \Rightarrow\left(a U^{(k)}+b V^{(k)}\right)|g\rangle=0 .
\end{aligned}
$$

Furthermore, since the $n^{\text {th }}$ mode of the product of operators $U^{(k)}$ and $V^{(l)}$ of $\operatorname{spin} k$ and $l$, respectively, is given by

$$
\left(U^{(k)}, V^{(l)}\right)_{n}=\sum_{q \geqq k+n} U_{n-q}^{(k)} V_{q}^{(l)}+\sum_{r \geqq-k+1} V_{n-r}^{(l)} U_{r}^{(k)}
$$


and the left-hand side is an operator of spin $k+l$, we have

(iii) $U^{(k)}|g\rangle=0, \quad V^{(l)}|g\rangle=0 \Rightarrow\left(U^{(k)}, V^{(l)}\right)|g\rangle=0$.

Lemma 5.1 together with (i), (ii), and (iii) proves the statement of this theorem.

Finally we show that the condition (5.15) becomes the vacuum condition of the $W_{p}$ algebra after the elimination of the variables $J_{n p}(n \in \mathbf{Z})$ which are redundant for the $p$-reduced KP hierarchy. In order to eliminate these variables, we first expand $S^{(k)}(\lambda)$ as a sum of operators with the usual normal ordering for the oscillators $J_{n}$. For example, we rewrite $S^{(k)}(\lambda)$ as

$$
\begin{aligned}
S^{(1)}(\lambda)= & \sum_{i=1}^{p} J\left(\lambda_{i}\right), \\
S^{(2)}(\lambda)= & \sum_{1 \leqq i<j \leqq p} J\left(\lambda_{i}\right) J\left(\lambda_{j}\right) \\
= & \sum_{1 \leqq i<j \leqq p}: J\left(\lambda_{i}\right) J\left(\lambda_{j}\right):+\sum_{1 \leqq i<j \leqq p}\left\langle J\left(\lambda_{i}\right) J\left(\lambda_{j}\right)\right\rangle, \\
S^{(3)}(\lambda)= & \sum_{1 \leqq i<j<k \leqq p} J\left(\lambda_{i}\right) J\left(\lambda_{j}\right) J\left(\lambda_{k}\right) \\
= & \sum_{1 \leqq i<j<k \leqq p}: J\left(\lambda_{i}\right) J\left(\lambda_{j}\right) J\left(\lambda_{k}\right):+\sum_{1 \leqq i<j<k \leqq p}\left\{\left\langle J\left(\lambda_{i}\right) J\left(\lambda_{j}\right)\right\rangle J\left(\lambda_{k}\right)\right. \\
& \left.+\left\langle J\left(\lambda_{i}\right) J\left(\lambda_{k}\right)\right\rangle J\left(\lambda_{j}\right)+\left\langle J\left(\lambda_{j}\right) J\left(\lambda_{k}\right)\right\rangle J\left(\lambda_{i}\right)\right\},
\end{aligned}
$$

where $::$ is the usual normal ordering for the $J_{n}$ 's $\left(\right.$ recall that $J(\lambda)=\frac{1}{p}$ $\left.\sum_{n} \lambda^{-n / p-1} J_{n}\right)$, and \langle\rangle is the usual vacuum expectation value, $\left\langle J_{n} J_{m}\right\rangle=n \delta_{n+m, 0} \theta(n)$. We then define $\mathbf{W}^{(k)}(\lambda)$ by formally setting $J_{n p}(n \in \mathbf{Z})$ to 0 in this normal ordered form of $S^{(k)}(\lambda)$. Note that $\mathbf{W}^{(1)}(\lambda)$ vanishes identically, because $S^{(1)}(\lambda)=\sum_{n} \lambda^{-n-1} J_{n p}$ contains only the variables to be dropped. As is shown in Appendix $\mathrm{C}$, these $\mathbf{W}^{(k)}(\lambda)$ 's are identified with the generators of the $W_{p}$ algebra with Virasoro central charge $c=p-1$ that is to be constructed from $\mathbf{Z}_{p}$-twisted free bosons. Furthermore we have

Lemma 5.3. The condition $S_{n}^{(k)}|g\rangle=0(k=1,2, \ldots, p ; n \geqq-k+1)$ is equivalent to

$$
\left\{\begin{array}{l}
J_{n p}|g\rangle=0 \quad(n \geqq 0) \\
\mathbf{W}_{n}^{(k)}|g\rangle=0 \quad(k=2, \ldots, p ; n \geqq-k+1) .
\end{array}\right.
$$

Proof. First we note that $S_{n}^{(k)}$ can be expanded with respect to $J_{n p}(n \in \mathbf{Z})$ as

$$
S_{n}^{(k)}=\mathbf{W}_{n}^{(k)}+\sum_{l=1}^{k} \sum_{n_{1}, \ldots, n_{l} \in \mathbf{Z}} C_{n-\left(n_{1}+\ldots+n_{l}\right)}^{(k-l)}\left[n_{1}, \ldots, n_{l}\right]: J_{n_{1} p} \ldots J_{n_{l} p}: .
$$

Here the operators $C_{n-\left(n_{1}+\ldots+n_{l}\right)}^{(k-l)}\left[n_{1}, \ldots, n_{l}\right]$ do not contain $J_{n p}(n \in \mathbf{Z})$, and are obtained from $S_{n}^{(k)}$ by repeatedly taking commutators with the $J_{n p}$, s $(n \in \mathbf{Z})$ and then setting $J_{n p}(n \in \mathbf{Z})$ to 0 :

$$
C_{n-\left(n_{1}+\ldots+n_{l}\right)}^{(k-l)}\left[n_{1}, \ldots, n_{l}\right]=\left.\mathrm{const}\left[J_{-n_{l} p},\left[\ldots,\left[J_{-n_{1} p}, S_{n}^{(k)}\right] \ldots\right]\right]\right|_{J_{n p}=0(n \in \mathbf{Z})}
$$

This implies that

$$
C_{n-\left(n_{1}+\ldots+n_{l}\right)}^{(k-l)}\left[n_{1}, \ldots, n_{l}\right]=\text { const } \mathbf{W}_{n-\left(n_{1}+\ldots+n_{l}\right)}^{(k-l)},
$$


because we have

$$
\left[J_{n p}, S_{m}^{(k)}\right]=\left[S_{n}^{(1)}, S_{m}^{(k)}\right]=(p+1-k) n S_{m+n}^{(k-1)},
$$

which follows from

$$
S^{(1)}\left(\lambda^{\prime}\right) S^{(k)}(\lambda)=\frac{p+1-k}{\left(\lambda^{\prime}-\lambda\right)^{2}} S^{(k-1)}(\lambda)+(\text { regular terms }) .
$$

The assertion follows immediately.

Thus combining Theorem 5.2 and Lemma 5.3, we obtain the following theorem:

Theorem 5.4. Let $\tau(x)$ be a $\tau$ function of the KP hierarchy that satisfies the conditions (4.9) and (4.10). Then the corresponding state $|g\rangle$ satisfies the conditions of the p-reduction

$$
J_{n p}|g\rangle=0 \quad(n \geqq 1)
$$

and the vacuum condition of the $W_{p}$ algebra

$$
\mathbf{W}_{n}^{(k)}|g\rangle=0 \quad(k=2,3, \ldots, p ; n \geqq-k+1) .
$$

By reinterpreting the oscillators $J_{n}$ 's as the differential operators $\mathscr{J}_{n}$ 's acting on $\tau(x)$ [see Eq. (4.32)], the set of Eqs. (5.29) and (5.30) are rewritten in the form of differential equations for $\tau(x)$. Thus we have seen that the statement conjectured in $[5,6]$ follows naturally from the infinite dimensional Grassmannian structure of 2D quantum gravity. ${ }^{7}$

\section{Conclusion}

In this paper, we showed that all the quantities of $2 \mathrm{D}$ quantum gravity can be expressed in terms of the infinite dimensional Grassmann manifold. Then, we found that every matrix model has the $W_{1+\infty}$ algebra as its fundamental symmetry, and that the $\tau$ function obeying the string equation satisfies the vacuum condition of the $W_{1+\infty}$ algebra. Furthermore, if we restrict ourselves to the $2 \mathrm{D}$ gravity coupled to $(p, q)$ conformal matters, then the vacuum condition of the $W_{1+\infty}$ algebra is reduced to the vacuum condition of the $W_{p}$ algebra with central charge $c=p-1$. In this sense, it seems that the real universal symmetry of $2 \mathrm{D}$ gravity is the $W_{1+\infty}$ algebra and that the $W_{p}$ algebra which appears in the $(p, q)$ quantum gravity is merely a by-product of this symmetry. Thus we expect that a deeper investigation of this structure will lead to a formalism in which we can control the renormalization group flows that correspond to the change of the parameter $p$. Furthermore, it might be useful for writing down the exact solutions of the nonperturbative $2 \mathrm{D}$ gravity coupled to the $c=1$ conformal matters. Among other interesting problems are the generalization to the $D$ - and $E$-type $W_{1+\infty}$ algebras and their application to $2 \mathrm{D}$ quantum gravity. Moreover, it has been reported recently that the relations among the orthogonal polynomials of matrix models are reduced to the Toda lattice hierarchy [13] (see also [14]). Their observations should also be understood in terms of infinite dimensional Grassmann manifolds.

${ }^{7}$ See [12] where some analyses are made based on Hirota's bilinear equation 


\section{Appendix A. Explicit Construction of the $\tau$ Functions}

In this appendix, we explain a method of constructing a $\tau$ function directly from a solution of the KP hierarchy.

\section{A.1. The KP Hierarchy and the Sato Equation}

In this subsection, we define the KP hierarchy and derive the Sato equation which plays an important role when we interpret the KP hierarchy as a dynamical system over an infinite dimensional Grassmann manifold [8-10].

First we introduce functions $u_{i}(t, x)(i=2,3, \ldots)$ of infinitely many variables $(t, x)=\left(t, x_{1}, x_{2}, x_{3}, \ldots\right)$ and define the Lax operator

$$
L \equiv \partial+u_{2}(t, x) \partial^{-1}+u_{3}(t, x) \partial^{-2}+\ldots
$$

as a pseudo-differential operator with respect to $\partial \equiv \partial / \partial t$. Here, $\partial^{-1}$ is defined so that $\partial \partial^{-1}=\partial^{-1} \partial=1$. Explicitly, we have for any function $f$

$$
\partial^{k} \cdot f=\sum_{l=0}^{\infty}\left(\begin{array}{l}
k \\
l
\end{array}\right) \frac{\partial^{l} f}{\partial t^{l}} \cdot \partial^{k-l},\left(\begin{array}{l}
k \\
l
\end{array}\right) \equiv \frac{k(k-1) \ldots(k-l+1)}{l !} .
$$

We further introduce the potentials $B_{n}$ and $B_{n}^{C}$ as

$$
B_{n} \equiv\left(L^{n}\right)_{+}, \quad B_{n}^{C} \equiv-\left(L^{n}\right)_{-},
$$

where ()$_{+}$(respectively ()$\left._{-}\right)$is non-negative (negative) power part of a pseudodifferential operator with respect to $\partial$. Then the KP hierarchy is defined as the set of the following differential equations:

$$
\frac{\partial}{\partial x_{n}} L=\left[B_{n}^{C}, L\right]\left(=\left[B_{n}, L\right]\right) .
$$

Note that they satisfy the integrability conditions $\partial^{2} L / \partial x_{n} \partial x_{m}=\partial^{2} L / \partial x_{m} \partial x_{n}$. The following theorem is fundamental.

Theorem 1. There exists a pseudo-differential operator $W$ of the form

$$
W=1+w_{1}(t, x) \partial^{-1}+w_{2}(t, x) \partial^{-2}+\ldots
$$

such that

$$
\begin{gathered}
L=W \partial W^{-1}, \\
\frac{\partial}{\partial x_{n}} W=B_{n}^{C} W=B_{n} W-W \partial^{n} .
\end{gathered}
$$

Equation (A.1.7) is called the Sato equation.

Proof. First we introduce a group $\mathscr{G}$ and its Lie algebra Lie $\mathscr{G}$ as the set of elements of the following forms:

$$
\begin{aligned}
& \mathscr{G} \equiv\left\{1+v_{1}(t) \partial^{-1}+v_{2}(t) \partial^{-2}+\ldots\right\}, \\
& \operatorname{Lie} \mathscr{G} \equiv\left\{b_{1}(t) \partial^{-1}+b_{2}(t) \partial^{-2}+\ldots\right\} .
\end{aligned}
$$

Then the $B_{n}^{C}$ 's can be regarded as the components of a Lie $\mathscr{G}$-valued 1-form $\Omega^{C}$ on the space of the $x_{n}$ 's:

$$
\Omega^{C} \equiv \sum_{n \geqq 1} B_{n}^{C} d x_{n}
$$


Then the KP Eq. (A.1.4) is rewritten as

$$
d L=\left[\Omega^{C}, L\right], \quad d \equiv \sum_{n \geqq 1} d x_{n} \frac{\partial}{\partial x_{n}} .
$$

Furthermore, by using an identity $\frac{\partial}{\partial x_{l}} B_{k}^{C}=\left(\left[B_{l}, B_{k}^{C}\right]\right)_{-}$, one can easily show that Eq. (A.1.10) leads to the zero-curvature condition

$$
d \Omega^{C}=\Omega^{C} \wedge \Omega^{C} \text {. }
$$

Thus the connection $\Omega^{C}$ is a pure gauge and can be written in the form

$$
\Omega^{C}=-V^{-1} d V
$$

where $V$ is a $\mathscr{G}$-valued function of the $x_{n}$ 's. It is also easy to see that $d\left(V L V^{-1}\right)=0$, which indicates that the $V L V^{-1}$ has the form

$$
V L V^{-1}=\partial+r_{2}(t) \partial^{-1}+r_{3}(t) \partial^{-2}+\ldots
$$

Therefore, $V L V^{-1}$ can be expressed as

$$
V L V^{-1}=U \partial U^{-1}
$$

using an element $U$ of $\mathscr{G}$ which depends only on $t$ :

$$
U=1+p_{1}(t) \partial^{-1}+p_{2}(t) \partial^{-2}+\ldots
$$

Hence, if we denote $V^{-1} U$ by $W$, that is,

$$
W \equiv V(t, x)^{-1} U(t) \equiv 1+w_{1}(t, x) \partial^{-1}+w_{2}(t, x) \partial^{-2}+\ldots,
$$

then the following relations hold:

$$
\begin{gathered}
L=W \partial W^{-1} \\
\Omega^{C}=-V^{-1} d V=-W d W^{-1}=d W \cdot W^{-1} .
\end{gathered}
$$

The latter equation $d W=\Omega^{C} W$ is nothing but the Sato equation

$$
\frac{\partial}{\partial x_{n}} W=B_{n}^{C} W=B_{n} W-W \partial^{n}
$$

Remark. From the identity $B_{1}=(L)_{+}=\partial / \partial t$, we obtain

$$
\frac{\partial}{\partial x_{1}} L=\left[B_{1}, L\right]=\frac{\partial}{\partial t} L .
$$

Thus every function $f$ appearing in the KP equation depends on the variables $(t, x)$ in the following manner:

$$
f=f\left(t+x_{1}, x_{2}, x_{3}, \ldots\right)
$$

so that we can (and will) set $t=0$ without loss of generality. Then $\partial$ is interpreted as a differential operator $\partial / \partial x_{1}$. 


\section{A.2. $\tau$ Functions and an Infinite Dimensional Grassmann Manifold}

In this subsection, by using the Sato equation, we show that the KP hierarchy is nothing but a dynamical system over an infinite dimensional Grassmann manifold [8-10]. Furthermore we explain that all the unknown functions $u_{i}(x)$ 's can be described in terms of a single function, Hirota's $\tau$ function [15]. In what follows, we assume that the functions $w_{j}(x)$ can be taylor-expanded around a point $x^{0}=\left(x_{1}^{0}, x_{2}^{0}, \ldots\right)$. In particular, we restrict ourselves to the case $x^{0}=0$. Generalization to the cases $x^{0} \neq 0$ is straightforward.

Let $H$ be the linear space consisting of pseudo-differential operators, which is isomorphic to $\mathbf{C}^{\mathbf{Z}}$.

$$
H=\left\{\sum_{k \in \mathbf{Z}} a_{-k-1 / 2} \partial^{k}\right\},
$$

and $H_{-}$be a linear subspace of $H$ consisting of all the differential operators. Then all the linear subspaces of $H$ which are linearly isomorphic to $H_{-}$make an infinite dimensional Grassmann manifold, and we denote it by UGM (Universal Grassmann Manifold). ${ }^{8}$

Now we make a mapping from the set of the solutions of the KP equation $\{W\}$ into a set of orbits in the UGM in the following way. First we construct the vectors $\eta^{(n)}(x)(n=0,1,2, \ldots)$ in $H$ as those whose components are the coefficients of the pseudo-differential operators $\partial^{n} W$ :

$$
\begin{gathered}
\partial^{n} W=\sum_{k \in \mathbf{Z}} \eta_{-k-1 / 2}^{(n)}(x) \partial^{k}, \\
\eta^{(n)}(x)=\left(\eta_{r}^{(n)}(x)\right)_{r \in \mathbf{Z}+1 / 2}=\left[\begin{array}{c}
\vdots \\
\eta_{3 / 2}^{(n)}(x) \\
\eta_{1 / 2}^{(n)}(x) \\
\eta_{-1 / 2}^{(n)}(x) \\
\eta_{-3 / 2}^{(n)}(x) \\
\vdots
\end{array}\right] .
\end{gathered}
$$

Then we define the subspace spanned by these vectors $\eta^{(0)}(x), \eta^{(1)}(x), \ldots$ and denote it by $V(x)$ :

$$
V(x)=\left[\eta^{(0)}(x), \eta^{(1)}(x), \ldots\right] .
$$

Note that we can represent the subspace $V(x)$ as a $\mathbf{Z} \times \mathbf{Z}_{+}$matrix

$$
\eta(x)=\left[\eta^{(0)}(x) \eta^{(1)}(x) \ldots\right] .
$$

Obviously, this matrix has an ambiguity of the right-multiplication of $G L\left(\mathbf{Z}_{+}\right)$, which corresponds to changes of the basis of $V(x)$.

The time (x) evolution of the $V(x)$ in UGM is, in general, determined from the Sato Eq. (A.1.7). This evolution can be represented in terms of the corresponding matrix in the following form:

$$
\eta(x)=e^{-\sum_{n \geqq 1} x_{n} \Lambda^{n}} \eta(0) C(x), \quad C(x) \in G L\left(\mathbf{Z}_{+}\right),
$$

${ }^{8}$ For more mathematically complete definitions, see [10] 
where $\Lambda=\left(\Lambda_{k l}\right)=\left(\delta_{k, l+1}\right) \in G L(\mathbf{Z})$. In fact, since the first term on the right-hand side of Eq. (A.1.7) has the form $B_{n} W=\sum_{j \geq 0} b_{n, j} \partial^{j} W$, it does not change the subspace $V(x)$ itself and gives rise to the factor $C(x)$ in Eq. (A.2.6). Hence $x$-evolution of the $V(x)$ comes only from the second term $-W \partial^{n}$ in Eq. (A.1.7), which is integrated into $\exp \left[-\sum_{n} x_{n} \Lambda^{n}\right]$ in the matrix representation. Thus we have seen that the KP hierarchy is nothing but a dynamical system over the UGM whose time evolution is given in a simple form $\exp \left[-\sum_{n} x_{n} \Lambda^{n}\right]$.

We next show that a solution of the $\mathrm{KP}$ equation, $W$, which has infinitely many unknown functions $w_{j}(x)$, can be expressed by a single function, $\tau$ function. First let us introduce a matrix $\tilde{\eta}(x)$, which also represents the subspace $V(x)$, as

$$
\tilde{\eta}(x) \equiv e^{-\sum_{n \geqq 1} x_{n} \Delta^{n}} \eta(0),
$$

and further we decompose it in the following way:

$$
\begin{gathered}
\tilde{\eta}(x)=\left[\begin{array}{l}
\tilde{\eta}_{+}(x) \\
\tilde{\eta}_{-}(x)
\end{array}\right], \\
\tilde{\eta}_{+}(x) \equiv\left(\eta_{r}^{(n)}(x)\right)_{r>0, n \geqq 0}, \quad \tilde{\eta}_{-}(x) \equiv\left(\eta_{r}^{(n)}(x)\right)_{r}<0, n \geqq 0 .
\end{gathered}
$$

Then the $\tau$ function corresponding to the subspace $V(x)$ is defined as

$$
\tau(x) \equiv \operatorname{det} \tilde{\eta}_{-}(x) .
$$

This $\tau$ function completely reproduces the solution of the KP equation due to the following theorem:

Theorem 2. Let $\tau(x)$ be the $\tau$ function which corresponds to a solution of the Sato equation, $W=\sum_{j \geqq 0} w_{j}(x) \partial^{-j}$. Then it holds that

$$
\sum_{j \geqq 0} w_{j}(x) k^{-j}=\tau\left(x-\varepsilon\left(k^{-1}\right)\right) / \tau(x),
$$

where $x-\varepsilon\left(k^{-1}\right)=\left(x_{1}-1 / k, x_{2}-1 /\left(2 k^{2}\right), x_{3}-1 /\left(3 k^{3}\right), \ldots\right)$.

Proof. First, if we rewrite $\tilde{\eta}(x)$ as

$$
\tilde{\eta}(x)=\left[\begin{array}{c}
\tilde{\eta}_{+}(x) \tilde{\eta}_{-}(x)^{-1} \\
1
\end{array}\right] \tilde{\eta}_{-}(x) \equiv \varrho(x) \tilde{\eta}_{-}(x),
$$

then the matrix $\varrho(x)$ also represents the subspace $V(x)$. Noting that the vector having the form

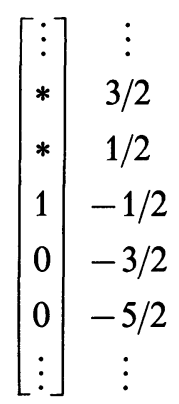


does not exist in $V(x)$ except for

$$
\eta^{(0)}(x)=\left[\begin{array}{c|c}
\vdots & \vdots \\
w_{2}(x) & 3 / 2 \\
w_{1}(x) & 1 / 2 \\
1 & -1 / 2 \\
0 & -3 / 2 \\
0 & -5 / 2 \\
\vdots & \vdots
\end{array}\right.
$$

we find that the first column vector of the matrix $\varrho(x)$ is nothing but $\eta^{(0)}(x)$. Next, if we calculate $\tilde{\eta}\left(x-\varepsilon\left(k^{-1}\right)\right)$, then we can show from Eqs. (A.2.6), (A.2.7), and (A.2.9) that

$$
\begin{aligned}
\tilde{\eta}\left(x-\varepsilon\left(k^{-1}\right)\right) & =\exp \left[\sum_{n \geqq 1} \frac{1}{n}\left(\frac{\Lambda}{k}\right)^{n}\right] \tilde{\eta}(x) \\
& =\sum_{j \geqq 0}\left(\frac{\Lambda}{k}\right)^{j} \varrho(x) \tilde{\eta}_{-}(x) \\
& \equiv\left[\begin{array}{l}
\tilde{\varrho}_{+}(x, k) \\
\tilde{\varrho}_{-}(x, k)
\end{array}\right] \tilde{\eta}_{-}(x) .
\end{aligned}
$$

Since a straightforward calculation shows that

$$
\operatorname{det} \tilde{\varrho}_{-}(x, k)=\sum_{j \geqq 0} w_{j}(x) k^{-j},
$$

we conclude that

$$
\begin{aligned}
\tau\left(x-\varepsilon\left(k^{-1}\right)\right) & =\operatorname{det} \tilde{\eta}_{-}\left(x-\varepsilon\left(k^{-1}\right)\right) \\
& =\operatorname{det}\left(\tilde{\varrho}_{-}(x, k) \cdot \tilde{\eta}_{-}(x)\right) \\
& =\left(\sum_{j \geqq 0} w_{j}(x) k^{-j}\right) \cdot \tau(x) .
\end{aligned}
$$

The set of Eqs. (A.1.5)-(A.1.7) and (A.2.11) yields useful formulas which express the second derivatives of $\ln \tau$ in terms of the pseudo-differential operator $L$ :

$$
\begin{aligned}
& \frac{\partial^{2}}{\partial x_{1} \partial x_{n}} \ln \tau=\left(L^{n}\right)_{-1}, \\
& \frac{\partial^{2}}{\partial x_{2} \partial x_{n}} \ln \tau=2\left(L^{n}\right)_{-2}+\frac{\partial}{\partial x_{1}}\left(L^{n}\right)_{-1}, \\
& \frac{\partial^{2}}{\partial x_{3} \partial x_{n}} \ln \tau=3\left(L^{n}\right)_{-3}+3 \frac{\partial}{\partial x_{1}}\left(L^{n}\right)_{-2}+\frac{\partial^{2}}{\partial x_{1}^{2}}\left(L^{n}\right)_{-1}+3 \frac{\partial^{2}}{\partial x_{1}^{2}} \ln \tau \cdot\left(L^{n}\right)_{-1}, \\
& \ldots \text { etc. },
\end{aligned}
$$

where the symbol ()$_{-k}$ stands for the coefficient of $\partial^{-k}$.

\section{A.3. Free Fermion Representation of $\tau$ Functions}

In this subsection, we describe the method of expressing $\tau$ functions in terms of free fermions. 
First, by using the correspondence between UGM and the set of all decomposable states [see Eqs. (2.5) and (2.6)], we construct a multi-fermion state $\left|g^{\prime}\right\rangle$ from the matrix $\eta(0)=\left[\eta^{(0)}(0) \eta^{(1)}(0) \ldots\right]$ which represents the subspace $V(0)$ :

$$
\left|g^{\prime}\right\rangle=\sum_{r_{0}, r_{1}, \ldots} \eta_{r_{0}}^{(0)}(0) \eta_{r_{1}}^{(1)}(0) \ldots \psi_{r_{0}}^{\dagger} \psi_{r_{1}}^{\dagger} \ldots|-\infty\rangle
$$

Then, the multi-fermion state corresponding to the matrix $\tilde{\eta}(x)=\exp \left[-\sum_{n} x_{n} \Lambda^{n}\right]$ $\eta(0)$ in Eq. (A.2.7) is

$$
\left|g^{\prime}(x)\right\rangle \equiv e^{-\sum_{n \geqq 1} x_{n} J_{n}}\left|g^{\prime}\right\rangle,
$$

where $J_{n}=\sum_{r}: \psi_{r-n}^{\dagger} \psi_{r}:(n \in \mathbf{Z})$. Thus

$$
\begin{aligned}
\tau(x) & =\operatorname{det} \tilde{\eta}_{-}(x)=\left\langle 0 \mid g^{\prime}(x)\right\rangle \\
& =\left\langle 0\left|e^{-\sum_{n \geq 1}^{\Sigma} x_{n} J_{n}}\right| g^{\prime}\right\rangle .
\end{aligned}
$$

Note that this state $\left|g^{\prime}\right\rangle$ can be expressed in terms of the Clifford group Cliff:

$$
\left|g^{\prime}\right\rangle=g^{\prime}|0\rangle, \quad g^{\prime} \in \text { Cliff },
$$

where the Clifford group is the set of the elements of the following form:

$$
\text { Cliff }=\left\{g=\exp \left[\sum_{r, s}: \psi_{r}^{\dagger} b_{r s} \psi_{s}:\right]\right\}
$$

Thus the $\tau(x)$ is also written as

$$
\tau(x)=\left\langle 0\left|e^{-\sum_{n \geq 1}^{\Sigma} x_{n} J_{n}} g^{\prime}\right| 0\right\rangle, \quad g^{\prime} \in \text { Cliff. }
$$

The above expression (A.3.3) has a different sign of exponent from that in Eq. (2.11). We can change the former by carrying out the $C P$ transformation

$$
\begin{aligned}
& \psi_{r} \mapsto \psi_{-r}^{\dagger}, \\
& \psi_{r}^{\dagger} \mapsto \psi_{-r} .
\end{aligned}
$$

In fact, under this mapping, $J_{n}$ changes its sign; $J_{n} \mapsto-J_{n}$, while the vacuum $|0\rangle$ remains unchanged. Moreover, recalling that $\tau$ functions can be calculated by using algebraic relations of fermions alone, we find that $\tau(x)$ is unchanged under the transformation. Thus, denoting the transformed element in Cliff by $g$, the $\tau(x)$ can be reexpressed as follows:

$$
\begin{aligned}
\tau(x) & =\left\langle 0\left|e^{-\sum_{n \geqq 1}^{\sum} x_{n} J_{n}} g^{\prime}\right| 0\right\rangle=\left\langle 0\left|e^{\sum_{n \geqq 1}^{\sum} x_{n} J_{n}} g\right| 0\right\rangle \\
& =\left\langle 0\left|e^{\sum_{n=1}^{\sum} x_{n} J_{n}}\right| g\right\rangle \quad(|g\rangle \equiv g|0\rangle) .
\end{aligned}
$$

\section{Appendix B. The Structure of $R\left[\left\{S^{(k)} ; k+1,2, \ldots, p\right\}\right]$}

In this appendix, by explicit calculations we check Lemma 5.1 for operators of spin up to 6 . 
First in order to simplify the calculations, we generalize the normal ordering $::$ defined in Sect. 5 as follows. If we consider a small disk $D$ on the single-sheeted $\lambda$ plane $(D \subset C-\{0\})$, it corresponds to $p$ disks $D_{1}, \ldots, D_{p}$ on the $p$-sheeted $\lambda$ plane. We denote by $\lambda_{i}$ the point in $D_{i}$ that corresponds to $\lambda \in D$. Furthermore we denote $J\left(\lambda_{i}\right)$ by $J_{i}(\lambda)$. We then define the normal ordering of $J_{i}(\lambda)$ as that of $p$ independent current operators on the complex $\lambda$ plane. For example, we have

$$
\begin{aligned}
& J_{i}(\lambda)=\circ^{\circ} J_{i}(\lambda) \circ \\
& J_{i}\left(\lambda^{\prime}\right) J_{j}(\lambda)=\frac{\delta_{i j}}{\left(\lambda^{\prime}-\lambda\right)^{2}}+{ }^{\circ} J_{i}\left(\lambda^{\prime}\right) J_{j}(\lambda) \circ \\
& J_{i}\left(\lambda^{\prime \prime}\right) J_{j}\left(\lambda^{\prime}\right) J_{k}(\lambda)=\frac{\delta_{i j}}{\left(\lambda^{\prime \prime}-\lambda^{\prime}\right)^{2}} J_{k}(\lambda) \\
& \quad+\frac{\delta_{i k}}{\left(\lambda^{\prime \prime}-\lambda\right)^{2}} J_{j}\left(\lambda^{\prime}\right)+\frac{\delta_{j k}}{\left(\lambda^{\prime}-\lambda\right)^{2}} J_{i}\left(\lambda^{\prime \prime}\right)+\circ J_{i}\left(\lambda^{\prime \prime}\right) J_{j}\left(\lambda^{\prime}\right) J_{k}(\lambda)^{\circ} .
\end{aligned}
$$

Clearly the normal ordering considered in Sect. 5 is a special case of this one.

Next we make some formulas which relate this normal ordering to the product defined by Eq. (5.9). Suppressing $\lambda$ and denoting the summation for $i$ by an overline as

$$
\overline{J^{l}\left(\partial\left(J^{q}\right)\right)^{m}\left(\partial^{2}\left(J^{r}\right)\right)^{n}}=\sum_{i=1}^{p} J_{i}(\lambda)^{l}\left(\partial\left(J_{i}(\lambda)^{q}\right)\right)^{m}\left(\partial^{2}\left(J_{i}(\lambda)^{r}\right)\right)^{n}
$$

we have the following formulas:

\section{Formula.}

(i) $\left.\left.\left.(\underbrace{S^{(1)},\left(S^{(1)},\left(S^{(1)}, \ldots\left(S^{(1)}\right.\right.\right.}_{l}, A) \ldots\right)\right)\right)=\circ S^{(1)^{l}} A \circ$ for any operator $A(\lambda)$,

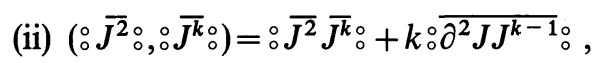

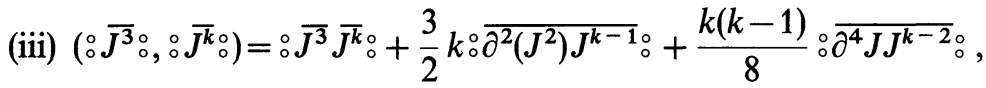

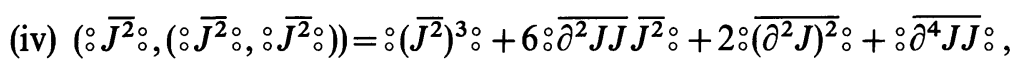

(v) $\left(\circ \bar{J}^{2} \circ, \circ \overline{\partial^{2} J J}\right)=\circ \overline{\left(\partial^{2} J\right)^{2}} \circ+\frac{1}{2} \circ \overline{\partial^{4} J J} \circ+\circ \overline{\partial^{2} J J} \overline{J^{2}} \circ$.

Proof. (i) First we consider $\left(S^{(1)}(\lambda), S^{(1)}(\lambda)\right)$. Recalling that $S^{(1)}(\lambda)=\sum_{i} J_{i}(\lambda)$, we have

$$
\begin{aligned}
S^{(1)}\left(\lambda^{\prime}\right) S^{(1)}(\lambda) & =\sum_{i} J_{i}\left(\lambda^{\prime}\right) \sum_{j} J_{j}(\lambda) \\
& =\sum_{i, j}\left(\frac{\delta_{i j}}{\left(\lambda^{\prime}-\lambda\right)^{2}}+\circ J_{i}\left(\lambda^{\prime}\right) J_{j}(\lambda)_{\circ}^{\circ}\right) \\
& =\frac{p}{\left(\lambda^{\prime}-\lambda\right)^{2}}+\circ S^{(1)}\left(\lambda^{\prime}\right) S^{(1)}(\lambda)^{\circ} .
\end{aligned}
$$

Taking the coefficient of $\left(\lambda^{\prime}-\lambda\right)^{0}$, we obtain

$$
\left(S^{(1)}(\lambda), S^{(1)}(\lambda)\right)=\circ S^{(1)}(\lambda) S^{(1)}(\lambda) \circ .
$$


Similarly for any operator $A(\lambda)$ we have

$$
\left(S^{(1)}(\lambda), A(\lambda)\right)=\circ S^{(1)}(\lambda) A(\lambda) \text { 。. }
$$

Using this equation repeatedly, we have the formula (i).

(ii) For example, we show the calculation for the simplest case, $k=2$. Following the definition of the normal ordering, we have

$$
\begin{aligned}
& \circ \sum_{i} J_{i}\left(\lambda^{\prime}\right)^{2 \circ} \circ \sum_{j} J_{j}(\lambda)^{2 \circ} \\
& =\sum_{i, j}\left(\frac{2 \delta_{i j}}{\left(\lambda^{\prime}-\lambda\right)^{4}}+\frac{4 \delta_{i j}}{\left(\lambda^{\prime}-\lambda\right)^{2}} \circ^{\circ} J_{i}\left(\lambda^{\prime}\right) J_{j}(\lambda)_{\circ}^{\circ}+\circ J_{i}\left(\lambda^{\prime}\right)^{2} J_{j}(\lambda)^{2} \circ\right) \text {. }
\end{aligned}
$$

Expanding this expression with respect to $\lambda^{\prime}$ around $\lambda$ and taking the coefficient of $\left(\lambda^{\prime}-\lambda\right)^{0}$, we have

$$
\left(\stackrel{\circ}{\circ} \sum_{i} J_{i}(\lambda)^{2 \circ} \circ \circ^{\circ} \sum_{j} J_{j}(\lambda)^{2 \circ} \circ\right)=2 \circ \sum_{i} \partial^{2} J_{i}(\lambda) J_{i}(\lambda) \circ+\circ \sum_{i} J_{i}(\lambda)^{2} \sum_{j} J_{j}(\lambda)^{2 \circ} \circ
$$

(iii) - (v) can be shown by similar calculations.

Using these formulas, we can rather easily check the statement of Lemma 5.1 for operators of lower spins. In the following we abbreviate $R\left[\left\{S^{(k)} ; k=1, \ldots, p\right\}\right]$ to $R^{(p)}$.

$\operatorname{spin} 1$

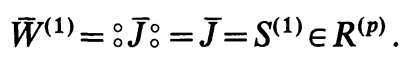

$\operatorname{spin} 2$

$$
\begin{aligned}
2 \bar{W}^{(2)} & =\circ \bar{J}^{2} \circ[\text { Eqs. }(5.1) \text { and }(5.6)] \\
& =\circ\left(S^{(1)}\right)^{2}-2 S^{(2)} \circ \\
& =\left(S^{(1)}, S^{(1)}\right)-2 S^{(2)}[\text { Formula (i) }] \in R^{(p)}
\end{aligned}
$$

$\operatorname{spin} 3$

$$
\begin{aligned}
3 \bar{W}^{(3)} & =\circ \bar{J}^{3} \circ[\text { Eqs. }(5.1) \text { and }(5.6)] \\
& =\circ\left(S^{(1)}\right)^{3}-3 S^{(1)} S^{(2)}+3 S^{(3)} \circ \\
& =\left(S^{(1)},\left(S^{(1)}, S^{(1)}\right)\right)-3\left(S^{(1)}, S^{(2)}\right)+3 S^{(3)}[\text { Formula }(i)] \in R^{(p)}
\end{aligned}
$$

$\operatorname{spin} 4$

It is easier to consider the following combination:

$$
4 \bar{W}^{(4)}+\frac{3}{5} \partial^{2} \bar{W}^{(2)}=\circ \bar{J}^{4}+\bar{\partial}^{2} J J^{\circ} \text { [Eqs. (5.1) and (5.6)]. }
$$

For the first term on the right-hand side, we have

$$
\circ \overline{J^{4}} \circ=\circ\left(S^{(1)}\right)^{4}-4\left(S^{(1)}\right)^{2} S^{(2)}+4 S^{(1)} S^{(3)}-4 S^{(4)}+2\left(S^{(2)}\right)^{2} \circ \text {. }
$$

By using Formula (i), it is obvious that each term on the right-hand side except for the last one belongs to $R^{(p)}$. Thus we have

$$
\circ \bar{J}^{4} \circ \equiv 2 \circ\left(S^{(2)}\right)^{2} \circ\left(\bmod R^{(p)}\right) \text {. }
$$


In order to evaluate this, we express $\left(S^{(2)}\right)^{2}$ as a combination of the $\overline{J^{k}}$,s as (note that $\left.\bar{J}=S^{(1)}\right)$

$$
2\left(S^{(2)}\right)^{2}=\frac{1}{2}\left(\overline{J^{2}}\right)^{2}-\left(S^{(1)}\right)^{2} \overline{J^{2}}+\frac{1}{2}\left(S^{(1)}\right)^{4} .
$$

Using Formulas (i) and (ii) for $k=2$, and recalling $\circ{\overline{J^{2}}}_{\circ} \circ \in R^{(p)}$ as shown above, we have

$$
2 \circ\left(S^{(2)}\right)^{2 \circ} \equiv-\circ \overline{\partial^{2} J J} \circ\left(\bmod R^{(p)}\right) .
$$

Thus we obtain

$$
\circ \bar{J}_{\circ}^{\circ} \equiv-\circ \overline{\partial^{2} J J} \circ\left(\bmod R^{(p)}\right),
$$

which indicates that the combination in Eq. (B.11) and hence $\bar{W}^{(4)}$ belongs to $R^{(p)}$. $\operatorname{spin} 5$

Here we note that as a set of basis of $W_{1+\infty}(p)$ we can take $\bar{P}^{(k)}(\lambda)=\sum_{i=1}^{p} P^{(k)}\left(\lambda_{i}\right)$ $(k=1,2, \ldots)$ instead of the $\bar{W}^{(k)}$ 's [see Eq. (5.1)]. Using Eqs. (5.1) and (5.2), we have $\bar{P}^{(5)}=\circ \bar{J}^{5}+\frac{5}{2}{\overline{J^{2}} \partial^{2} J_{\circ}^{\circ}}_{+}+\left(\right.$a linear combination of $\partial \bar{W}^{(4)}, \partial^{2} \bar{W}^{(3)}, \partial^{3} \bar{W}^{(2)}$ and $\left.\partial^{4} \bar{W}^{(1)}\right)$, which indicates the following equivalence assuming $\bar{W}^{(k)} \in R^{(k)}$ for $k=1,2,3,4$ :

$$
\bar{W}^{(5)} \in R^{(p)} \Leftrightarrow \bar{P}^{(5)} \in R^{(p)} \Leftrightarrow \circ \overline{J^{5}}+\frac{5}{2} \overline{J^{2} \partial^{2} J^{\circ}} \in R^{(p)} .
$$

In order to evaluate $\circ \bar{J}^{5} \circ$, we express $\overline{J^{5}}$ in terms of the elementary symmetric polynomials $S^{(k)}$ as

$$
\begin{aligned}
\circ \bar{J}_{\circ}^{5}= & \circ\left(S^{(1)}\right)^{5}-5\left(S^{(1)}\right)^{3} S^{(2)}+5\left(S^{(1)}\right)^{2} S^{(3)}-5 S^{(1)} S^{(4)}+5 S^{(5)} \\
& +5 S^{(1)}\left(S^{(2)}\right)^{2}-5 S^{(2)} S^{(3)} \circ .
\end{aligned}
$$

By using Formula (i), it is apparent that each term on the right-hand side except for the last two terms belongs to $R^{(p)}$. Thus we have

$$
\circ \bar{J}^{5} \circ \equiv 5 \circ S^{(1)}\left(S^{(2)}\right)^{2}-S^{(2)} S^{(3)} \circ\left(\bmod R^{(p)}\right) \text {. }
$$

We then express the right-hand side in terms of the $\bar{J}^{k}$ s as

$$
5 \circ S^{(1)}\left(S^{(2)}\right)^{2}-S^{(2)} S^{(3)} \circ=\frac{5}{6} \circ \overline{J^{2}} \overline{J^{3}}-\left(S^{(1)}\right)^{2} \overline{J^{3}}-\left(S^{(1)}\right)^{3} \overline{J^{2}}+\left(S^{(1)}\right)^{5 \circ} \text {. }
$$

Using Formula (i) and recalling that ${ }_{\circ}{\overline{J^{2}}}_{\circ} \circ \in R^{(p)}$ and ${ }_{\circ}{\overline{J^{3}}}_{\circ} \in R^{(p)}$ as shown above, we see that each term on the right-hand side except for the first one belongs to $R^{(p)}$. Furthermore, as for the first term the use of Formula (ii) for $k=2$ gives

$$
\begin{aligned}
\frac{5}{6} \circ{\overline{J^{2}}}^{J^{3}} \circ & =\frac{5}{6}\left(\circ{\overline{J^{2}}}_{\circ} \circ, \circ \overline{J^{3}} \circ\right)-\frac{5}{2} \circ \overline{\partial^{2} J J^{2}} \circ \\
& \equiv-\frac{5}{2} \circ \bar{\partial}^{2} J J^{2} \circ\left(\bmod R^{(p)}\right) .
\end{aligned}
$$

Therefore we find

$$
5 \circ S^{(1)}\left(S^{(2)}\right)^{2}-S^{(2)} S^{(3)} \circ \equiv-\frac{5}{2} \circ \overline{\partial^{2} J J^{2}} \circ\left(\bmod R^{(p)}\right),
$$

which proves that the combination $\circ \overline{J^{5}}+\frac{5}{2} \bar{J}^{2} \partial^{2} J_{\circ}^{\circ}$ and hence $\bar{W}^{(5)}$ belongs to $R^{(p)}$. spin 6

Using Eqs. (5.1) and (5.2), we can show that an appropriate linear combination of $\bar{W}^{(6)}, \partial \bar{W}^{(5)}, \partial^{2} \bar{W}^{(4)}, \ldots, \partial^{5} \bar{W}^{(1)}$ gives the following operator

$$
\bar{V}^{(6)}=\circ \overline{J^{6}}+3 \overline{\partial^{2} J J^{3}}+\frac{3}{2} \overline{\partial^{2}\left(J^{2}\right) J^{2}}+\frac{1}{2} \overline{\left(\partial^{2} J\right)^{2}}+\frac{1}{2} \overline{\partial^{4} J J^{\circ}} .
$$


Therefore the two statements $\bar{V}^{(6)} \in R^{(p)}$ and $\bar{W}^{(6)} \in R^{(p)}$ are equivalent under the assumption $\bar{W}^{(k)} \in R^{(p)}(k=1,2, \ldots, 5)$. Here we will show the former statement, since it is practically easier. In order to evaluate ${ }_{\circ} \bar{J}_{\circ}^{6}$, we express $\bar{J}^{6}$ in terms of the elementary symmetric polynomials $S^{(k)}$ as

$$
\begin{gathered}
\quad \circ \bar{J}_{\circ}^{\circ}=\circ A_{\circ}+\circ B_{\circ}^{\circ}, \\
A=\left(S^{(1)}\right)^{6}-6\left(S^{(1)}\right)^{4} S^{(2)}+6\left(S^{(1)}\right)^{3} S^{(3)}-6\left(S^{(1)}\right)^{2} S^{(4)}+6 S^{(1)} S^{(5)}-6 S^{(6)}, \\
B=9\left(S^{(1)}\right)^{2}\left(S^{(2)}\right)^{2}-12 S^{(1)} S^{(2)} S^{(3)}-2\left(S^{(2)}\right)^{3}+6 S^{(2)} S^{(4)}+3\left(S^{(3)}\right)^{2} .
\end{gathered}
$$

Since each term of $A$ contains operators $S^{(k)}$ other than $S^{(k)}$ other than $S^{(1)}$ at most linearly, Formula (i) indicates that $: A \circ$ belongs to $R^{(p)}$. We then express $B$ in terms of $\bar{J}^{\bar{k}}$ s as

$$
\begin{aligned}
B & =B_{1}+B_{2}+B_{3}+B_{4}, \\
B_{1} & =\frac{1}{3}\left(\overline{J^{3}}\right)^{2} \\
B_{2} & =-\frac{1}{8}\left(\overline{J^{2}}\right)^{3}+\frac{3}{4} \overline{J^{2}} \overline{J^{4}} \\
B_{3} & =\frac{3}{8}\left(\left(\overline{J^{2}}\right)^{2}-2 \overline{J^{4}}\right)\left(S^{(1)}\right)^{2}, \\
B_{4} & =-\frac{2}{3} \bar{J}^{3}\left(S^{(1)}\right)^{3}-\frac{9}{8} \overline{J^{2}}\left(S^{(1)}\right)^{4}+\frac{29}{24}\left(S^{(1)}\right)^{6} .
\end{aligned}
$$

Obviously $: B_{4} \circ$ belongs to $R^{(p)}$ because of Formula (i) (recall that $\circ \bar{J}^{2} \circ$, $\left.\circ \bar{J}_{\circ}^{3} \circ R^{(p)}\right)$. By using Formula (ii) for $k=2, \circ B_{3}^{\circ} \circ$ is written as

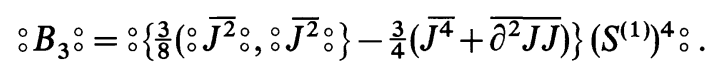

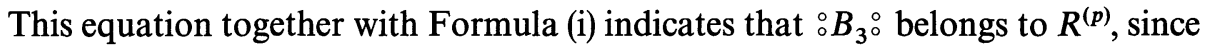
we have

$$
: \bar{J}_{\circ}^{2} \in R^{(p)} \quad \text { and } \quad: \bar{J}^{4}+{\overline{\partial^{2} J J}}_{\circ} \in R^{(p)}
$$

The evaluation of ${ }_{\circ}^{\circ} B_{2}^{\circ}$ is slightly nontrivial. Using the formulas (ii), (iv), and (v), we have

$$
\begin{aligned}
& \circ\left(\overline{J^{2}}\right)^{3 \circ} \circ=\left(\circ \bar{J}^{2} \circ,\left(\circ \bar{J}^{2} \circ, \circ \bar{J}_{\circ}^{2} \circ\right)\right)-6 \circ \overline{\partial^{2} J J} \bar{J}_{\circ} \circ \\
& -2 \circ \overline{\left(\partial^{2} J\right)^{2}} \circ-\circ \overline{\partial^{4} J J} \circ \text { [Formula (iv)], } \\
& \left.\circ{\overline{J^{2}}}^{4} \bar{J}_{\circ}=\left(\stackrel{\circ}{\circ}{\overline{J^{2}}}_{\circ}^{\circ}, \circ{\overline{J^{4}}}_{\circ}^{\circ}\right)-4 \stackrel{\circ}{\circ} \overline{\partial^{2} J J^{3}} \circ \text { 。 [Formula (ii) for } k=4\right]
\end{aligned}
$$

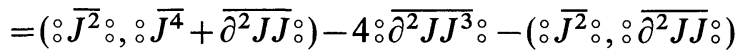

$$
\begin{aligned}
& =\left(\circ{\overline{J^{2}}}_{\circ}^{\circ}, \circ \bar{J}^{4}+{\overline{\partial^{2} J J}}_{\circ}\right)-4 \circ \bar{\partial}^{2} J J^{3} \circ \\
& \left.-\circ \overline{\left(\partial^{2} J\right)^{2}} \circ-\frac{1}{2} \circ \bar{\partial}^{4} J J_{\circ}^{\circ}-\circ \bar{J}^{2}{\overline{\partial^{2} J J}}_{\circ}^{\circ} \text { [Formula }(\mathrm{v})\right] \text {. }
\end{aligned}
$$

Therefore we have

$$
\begin{aligned}
& \circ B_{2} \circ=-\frac{1}{8}\left(\circ \bar{J}^{2} \circ,\left(\circ \bar{J}^{2} \circ, \circ \bar{J}^{2} \circ\right)\right)+\frac{3}{4}\left(\circ \bar{J}^{2} \circ, \circ \bar{J}^{4}+\overline{\partial^{2} J J_{\circ}^{\circ}}\right)
\end{aligned}
$$

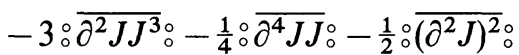

$$
\begin{aligned}
& \equiv-3 \circ \overline{\partial^{2} J J^{3}} \circ-\frac{1}{4} \circ \overline{\partial^{4} J J} \circ-\frac{1}{2} \circ \overline{\left(\partial^{2} J\right)^{2}} \circ\left(\bmod R^{(p)}\right) \text {. }
\end{aligned}
$$


Finally $: B_{1} \circ$ can be evaluated by using Formula (iii) for $k=3$ as

$$
\begin{aligned}
\circ B_{1} \circ & =\frac{1}{3} \circ\left(\overline{J^{3}}\right)^{2} \circ \\
& =\frac{1}{3}\left(\circ \bar{J}^{3} \circ, \circ \bar{J}^{3} \circ\right)-\frac{3}{2} \circ \overline{\partial^{2}\left(J^{2}\right) J^{2}} \circ-\frac{1}{4} \circ \overline{\partial^{4} J J} \circ \\
& \equiv-\frac{3}{2} \circ \overline{\partial^{2}\left(J^{2}\right) J^{2}} \circ-\frac{1}{4} \circ \overline{\partial^{4} J J} \circ\left(\bmod R^{(p)}\right),
\end{aligned}
$$

where the last equation holds because $\circ \bar{J}^{3} \circ$ belongs to $R^{(p)}$. Thus, combining Eqs. (B.24), (B.30), and (B.31), we find that $\bar{V}^{(6)}$ and hence $\bar{W}^{(6)}$ belongs to $R^{(p)}$.

Although we have written the equations with taking $\bmod R^{(p)}$, the above procedure gives explicit expressions of the $\bar{W}^{(k)}$ 's in terms of the $S^{(k)}$ 's as is shown in Eq. (5.13). Since these equations have such structures as

$$
\bar{W}^{(k)}=(-1)^{k+1} S^{(k)}+\left(\text { terms consisting of } S^{(l)} \text { 's with } l<k\right),
$$

we can easily obtain the inverse expression, that is,

$$
S^{(k)}=(-1)^{k+1} \bar{W}^{(k)}+\left(\text { terms consisting of } \bar{W}^{(l)} \text { 's with } l<k\right) .
$$

Thus we have checked Lemma 5.1 for operators of spin $k \leqq 6$

\section{Appendix C. Construction of the $W_{p}$ Algebra from $Z_{p}$-Twisted Bosons}

In this appendix, we show that the $\mathbf{W}^{(k)}(\lambda)$ 's defined in Sect. 5 can be identified with the generators of the $W_{p}$ algebra constructed from $\mathbf{Z}_{p}$-twisted bosons.

Let $\vec{\Phi}(\lambda)=\left(\Phi_{1}(\lambda), \ldots, \Phi_{p-1}(\lambda)\right)$ be a $(p-1)$-component vector consisting of free scalar fields $\Phi_{j}(\lambda)$ on the complex $\lambda$ plane. When $\Phi_{j}(\lambda)(j=1,2, \ldots, p-1)$ satisfies the untwisted boundary condition $\left(\Phi_{j}\left(e^{2 \pi i} \lambda\right)=\Phi_{j}(\lambda)\right)$, the generators $\mathbf{W}^{(k)}(\lambda)$ of the $W_{p}$ algebra with Virasoro central charge $c=p-1$ are constructed through the quantum Miura transformation [16]:

$$
\mathbf{W}^{(k)}(\lambda)=\sum_{n} \mathbf{W}_{n}^{(k)} \lambda^{-n-k}=(-1)^{k-1} \sum_{1 \leqq j_{1}<\ldots<j_{k} \leqq p}: \prod_{m=1}^{k}\left(\vec{h}_{j_{m}} \cdot \partial \vec{\Phi}(\lambda)\right): .
$$

(We took the limit $\alpha_{0} \rightarrow 0$ in Eqs. (2.7) and (2.9) in [16].) Here the $\vec{h}_{j}$ 's are the weight vectors of the fundamental representation of $\operatorname{sl}(p)\left(\right.$ in $\left.\mathbf{R}^{p-1}\right)$ and satisfy the relations

$$
\sum_{j=1}^{p} \vec{h}_{j}=0, \quad \vec{h}_{i} \cdot \vec{h}_{j}=\delta_{i j}-\frac{1}{p}
$$

The simple roots $\vec{\alpha}_{1}, \ldots, \vec{\alpha}_{p-1}$ of $s l(p)$ are given by

$$
\vec{\alpha}_{j}=\vec{h}_{j}-\vec{h}_{j+1} \text {. }
$$

It is known that the $\mathbf{W}^{(k)}(\lambda)$ 's in Eq. (C.1) generate the $W_{p}$ algebra [17].

In what follows, we will impose on the $\Phi_{j}(\lambda)$ a boundary condition which generates a cyclic permutation of the simple roots $\vec{\alpha}_{1}, \ldots, \vec{\alpha}_{p-1}$ and the lowest root $\vec{\alpha}_{0} \equiv-\sum_{j=1}^{p-1} \vec{\alpha}_{j}$, that is,

$$
\vec{h}_{j} \cdot \vec{\Phi}\left(e^{2 \pi i} \lambda\right)=\vec{h}_{j+1} \cdot \vec{\Phi}(\lambda) \quad(j=1,2, \ldots, p) \quad\left(\vec{h}_{p+1} \equiv \vec{h}_{1}\right) .
$$

We note that the $\mathbf{W}^{(k)}(\lambda)$ in Eq. (C.1) is single-valued under this boundary condition. 
The boundary condition (C.4) is diagonalized for the following scalar fields

$$
\phi_{l}(\lambda) \equiv \frac{1}{\sqrt{p}} \sum_{j=1}^{p} e^{2 \pi i j l / p} \vec{h}_{j} \cdot \vec{\Phi}(\lambda) \quad(l=1,2, \ldots, p-1),
$$

which satisfies a $\mathbf{Z}_{p}$-twisted boundary condition

$$
\phi_{l}\left(e^{2 \pi i} \lambda\right)=e^{2 \pi i l / p} \phi_{l}(\lambda) .
$$

Then the spin $k$ current $\mathbf{W}^{(k)}(\lambda)$ in Eq. (C.1) can be written in terms of $\phi_{l}(\lambda)$ by the formula

$$
\begin{aligned}
\mathbf{W}^{(k)}(\lambda)= & (-1)^{k-1} \sum_{1 \leqq j_{1}<\ldots<j_{k} \leqq p}: \prod_{m=1}^{k}\left[\frac{1}{\sqrt{p}} \sum_{l=1}^{p-1} e^{-2 \pi i l j_{m} / p} \partial \phi_{l}(\lambda)\right]: \\
& +\left(\text { terms proportional to } \lambda^{-2}, \lambda^{-4}, \ldots\right) .
\end{aligned}
$$

Here the second terms on the right-hand side are necessary in order to make the $\mathbf{W}^{(k)}(\lambda)$ 's close under the operator product expansion. It is easy to see that the $\mathbf{W}^{(k)}$ 's defined by this equation are identical to the $\mathbf{W}^{(k)}$ 's defined in Sect. 5 up to overall normalizations if we identify $\partial \phi_{l}(\lambda)$ with $\sum_{n=-l(\bmod p)} \frac{1}{\sqrt{p}} \lambda^{-n / p-1} J_{n}$. Note that the construction of the $W^{(k)}$ 's in Sect. 5 fixes the form of the second terms in Eq. (C.7) automatically without any ambiguity. Their explicit forms for $k \leqq 4$ are

$$
\begin{aligned}
& \mathbf{W}^{(2)}(\lambda)=\frac{1}{2} \sum_{l=1}^{p-1}: \partial \phi_{l}(\lambda) \partial \phi_{p-l}(\lambda):+\frac{p^{2}-1}{24 p} \frac{1}{\lambda^{2}},
\end{aligned}
$$

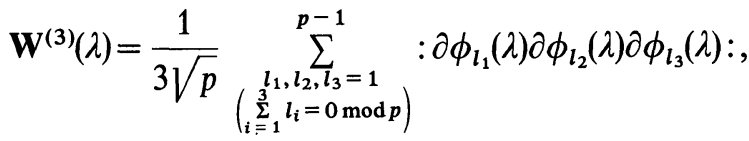

$$
\begin{aligned}
& \mathbf{W}^{(4)}(\lambda)=\frac{1}{4 p} \sum_{\substack { l_{1}, l_{2}, l_{3}, l_{4}=1 \\
\begin{subarray}{c}{1 \\
\sum \\
i=1{ l _ { 1 } , l _ { 2 } , l _ { 3 } , l _ { 4 } = 1 \\
\begin{subarray} { c } { 1 \\
\sum \\
i = 1 } }\end{subarray}}^{p-1}: \partial \phi_{l_{1}}(\lambda) \partial \phi_{l_{2}}(\lambda) \partial \phi_{l_{3}}(\lambda) \partial \phi_{l_{4}}(\lambda): \\
& -\frac{1}{8}:\left[\sum_{l=1}^{p-1} \partial \phi_{l}(\lambda) \partial \phi_{p-l}(\lambda)\right]^{2}: \\
& -\frac{1}{\lambda^{2}}\left[\frac{(p-6)\left(p^{2}-1\right)}{48 p^{2}} \sum_{l=1}^{p-1}: \partial \phi_{l}(\lambda) \partial \phi_{p-l}(\lambda):\right. \\
& \left.+\frac{1}{4} \sum_{l=1}^{p-1} \frac{1}{p}\left(1-\frac{l}{p}\right): \partial \phi_{l}(\lambda) \partial \phi_{p-l}(\lambda):\right] \\
& -\frac{1}{\lambda^{4}} \frac{1}{5760 p^{3}}\left(p^{2}-1\right)(p-2)(p-3)(5 p+7) \text {. }
\end{aligned}
$$

By using the contraction rule

$$
\begin{aligned}
\partial \phi_{k}\left(\lambda^{\prime}\right) \partial \phi_{l}(\lambda)= & \frac{\left(1-\frac{k}{p}\right)\left(\frac{\lambda^{\prime}}{\lambda}\right)^{k / p}+\left(\frac{k}{p}\right)\left(\frac{\lambda}{\lambda^{\prime}}\right)^{1-k / p}}{\left(\lambda^{\prime}-\lambda\right)^{2}} \delta_{k+l, p} \\
& +(\text { regular terms) },
\end{aligned}
$$


one can explicitly check the operator product expansion, which actually forms the $W_{p}$ algebra with central charge $c=p-1$ :

$$
\begin{aligned}
\mathbf{W}^{(2)}\left(\lambda^{\prime}\right) \mathbf{W}^{(2)}(\lambda)= & \frac{(p-1) / 2}{\left(\lambda^{\prime}-\lambda\right)^{4}}+\frac{2}{\left(\lambda^{\prime}-\lambda\right)^{2}} \mathbf{W}^{(2)}(\lambda)+\frac{1}{\lambda^{\prime}-\lambda} \partial_{\lambda} \mathbf{W}^{(2)}(\lambda)+\ldots, \\
\mathbf{W}^{(2)}\left(\lambda^{\prime}\right) \mathbf{W}^{(3)}(\lambda)= & \frac{3}{\left(\lambda^{\prime}-\lambda\right)^{2}} \mathbf{W}^{(3)}(\lambda)+\frac{1}{\lambda^{\prime}-\lambda} \partial_{\lambda} \mathbf{W}^{(3)}(\lambda)+\ldots, \\
\mathbf{W}^{(2)}\left(\lambda^{\prime}\right) \mathbf{W}^{(4)}(\lambda)= & \frac{(p-2)(p-3)}{2 p} \frac{1}{\left(\lambda^{\prime}-\lambda\right)^{4}} \mathbf{W}^{(2)}(\lambda) \\
& +\frac{4}{\left(\lambda^{\prime}-\lambda\right)^{2}} \mathbf{W}^{(4)}(\lambda)+\frac{1}{\lambda^{\prime}-\lambda} \partial_{\lambda} \mathbf{W}^{(4)}(\lambda)+\ldots, \\
\mathbf{W}^{(3)}\left(\lambda^{\prime}\right) \mathbf{W}^{(3)}(\lambda)= & \frac{2(p-1)(p-2)}{3 p} \frac{1}{\left(\lambda^{\prime}-\lambda\right)^{6}} \\
& +\frac{4(p-2)}{p} \frac{1}{\left(\lambda^{\prime}-\lambda\right)^{4}} \mathbf{W}^{(2)}(\lambda)+\frac{2(p-2)}{p} \frac{1}{\left(\lambda^{\prime}-\lambda\right)^{3}} \partial_{\lambda} \mathbf{W}^{(2)}(\lambda) \\
+ & \frac{1}{\left(\lambda^{\prime}-\lambda\right)^{2}}\left[4 \mathbf{W}^{(4)}(\lambda)+\frac{2(p-2)}{p}\left(\mathbf{W}^{(2)}(\lambda), \mathbf{W}^{(2)}(\lambda)\right)\right] \\
+ & \frac{1}{\lambda^{\prime}-\lambda} \partial_{\lambda}\left[2 \mathbf{W}^{(4)}(\lambda)\right. \\
+ & \left.\frac{p-2}{p}\left(\mathbf{W}^{(2)}(\lambda), \mathbf{W}^{(2)}(\lambda)\right)-\frac{p-2}{6 p} \partial_{\lambda}^{2} \mathbf{W}^{(2)}(\lambda)\right]+\ldots,
\end{aligned}
$$

Acknowledgements. We would like to thank S. Hosono, Y. Okamoto, and T. Sano for discussions.

\section{References}

1. Brézin, E., Kazakov, V.: Phys. Lett. B 236, 144 (1990)

Douglas, M., Shenker, S.: Nucl. Phys. B 335, 635 (1990)

Gross, D.J., Migdal, A.: Phys. Rev. Lett. 64, 127 (1990)

2. Gross, D.J., Migdal, A.: Nucl. Phys. B 340, 333 (1990)

3. Banks, T., Douglas, M., Seiberg, N., Shenker, S.: Phys. Lett. B 238, 279 (1990)

4. Douglas, M.: Phys. Lett. B 238, 176 (1990)

5. Fukuma, M., Kawai, H., Nakayama, R.: Int. J. Mod. Phys. A 6, 1385 (1991)

6. Dijkgraaf, R., Verlinde, E., Verlinde, H.: Loop equations and Virasoro constraints in nonperturbative 2-D quantum gravity. IAS/Princeton preprint IASSNS-HEP-90/48, PUPT1184 (1990)

7. Pope, C., Romans, L., Shen, X.: Nucl. Phys. B 339, 191 (1990); Phys. Lett. B 242, 401 (1990); B 245, 72 (1990)

Bergshoeff, E., Pope, C., Romans, L., Sezgin, E., Shen, X.: Phys. Lett. B 245, 447 (1990)

8. Sato, M.: Soliton equations as dynamical systems on infinite dimensional Grassmann manifolds. RIMS Kokyuroku 439, 30 (1981)

9. Date, E., Jimbo, M., Kashiwara, M., Miwa, T.: Transformation groups for soliton equations. In: Proc. of RIMS Symposium on non-linear integrable systems-classical theory and quantum theory. Kyoto, Japan, May 1981, Jimbo, M., Miwa, T. (eds.). Singapore: World Scientific Publishing Co. 1983

10. Segal, G., Wilson, G.: Pub. Math. IHES 61, 5 (1985) 
11. Witten, E.: Nucl. Phys. B 340, 281 (1990)

Distler, J.: Nucl. Phys. B 342, 523 (1990)

Dijkgraaf, R., Witten, E.: Nucl. Phys. B 342, 486 (1990)

Verlinde, E., Verlinde, H.: A solution of two dimensional topological quantum gravity. IAS preprint IASSNS-HEP-90/40 (1990)

$\mathrm{Li}$, K.: Recursion relations in topological gravity with minimal matter. Caltech preprint, CALT-68-1670 (1990)

12. Yoneya, T.: Action principle, Virasoro structure and analycity in non-perturbative twodimensional gravity. Tokyo preprint, UT-Komaba 90-28 (1990)

Goeree, J.: W constraints in 2-D quantum gravity. Utrecht preprint, THU-19 (1990)

13. Gerasimov, A., Marshakov, A., Mironov, A., Morozov, A., Orlov, A.: Matrix models of 2D gravity and toda theory. Lebedev Inst. preprint (1990)

Martinec, E.: On the origin of integrability in matrix models, Chicago preprint, EFI-90-67 (1990)

Alvarez-Gaumé, L., Gomez, C., Lacki, J.: Integrability in random matrix models. CERN preprint, CERN-TH. 5875/90 (1990)

14. Itoyama, H., Matsuo, Y.: Noncritical Virasoro algebra of $d<1$ matrix model and quantized string field. ITP preprint, ITP-SB-90-75 (1990)

15. Hirota, R.: Direct method of finding exact solutions of nonlinear evolution equations. Springer Lecture Notes in Math. 515, 40 (1976)

16. Fateev, V., Lykyanov, S.: Int. J. Mod. Phys. A 3, 507 (1988)

17. Lykyanov, S.: Funct. Anal. Appl. 22, 255 (1988)

Communicated by H. Araki 
